\title{
Logarithmic del Pezzo surfaces of rank one with unique singular points
}

\author{
By Hideo KoJima* \\ (Received March 25, 1998) \\ (from Osaka Journal of Mathematics)
}

\section{Introduction}

Let $k$ be an algebraically closed field of characteristic zero. Let $\bar{X}$ be a normal algebraic surface with a unique quotient singular point $P$. Let $\pi: X \rightarrow \bar{X}$ be a minimal resolution of $\bar{X}$ and let $D=\sum_{i=1}^{n} D_{i}$ be the reduced exceptional divisor with respect to $\pi$, where the $D_{i}$ are irreducible components. Then there exists uniquely an effective Q-divisor $D^{\#}=\sum_{i=1}^{n} \alpha_{i} D_{i}$ such that $D^{\#}+K_{X}$ is numerically equivalent to $\pi^{*}\left(K_{\bar{X}}\right)$. Since $P$ is a log terminal singular point (for the definition, see [3]), it follows that $0 \leq \alpha_{i}<1$ for any $i$. $\operatorname{Put} \operatorname{Bk}(D)=D-D^{\#}$.

Definition. The above pair $(X, D)$ is almost minimal if, for every irreducible curve $C$, either $\left(D^{\#}+K_{X} \cdot C\right) \geq 0$ or the intersection matrix of $C+\mathrm{Bk}(D)$ is not negative definite. The singular point $P$ is almost minimal in $\bar{X}$ if the pair $(X, D)$ is almost minimal.

Starting with arbitrary quotient singular points, we can construct almost minimal quotient singular points, though the singularities might be changed from the original ones.

In the present article, we assume that a pair $(X, D)$ is almost minimal and the logarithmic Kodaira dimension $\bar{\kappa}(X-D)=-\infty$. Since $K_{\bar{X}}$ is then not numerically effective, there exists an extremal rational curve $\bar{\ell}$ on $\bar{X}$. Let $\ell$ be the proper transform of $\bar{\ell}$ on $X$. By Miyanishi-Tsunoda [7, Lemma 2.7], one of the following two cases then takes place:

(A) The intersection matrix of $\ell+\mathrm{Bk}(D)$ is negative semi-definite, but not negative definite. Furthermore, $\left(\bar{\ell}^{2}\right)=0$.

(B) The Picard number $\rho(\bar{X})$ equals 1 , and $-K_{\bar{X}}$ is ample. Namely, $(X, D)$ is a logarithmic del Pezzo surface of rank one with contractible boundary (henceforth

*The author is partially supported by JSPS Research Fellowships for Young Scientists and Grant-in-Aid for Scientific Research, the Japanese Ministry of Education, Science, Sports and Culture. 

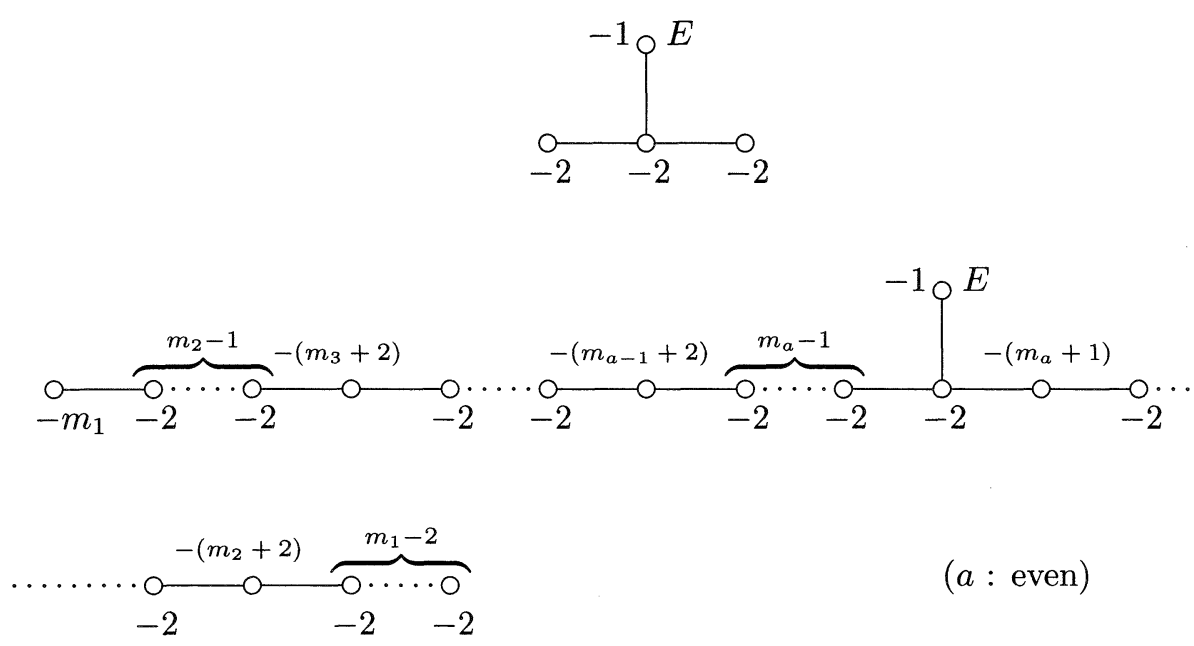

$(a:$ even $)$
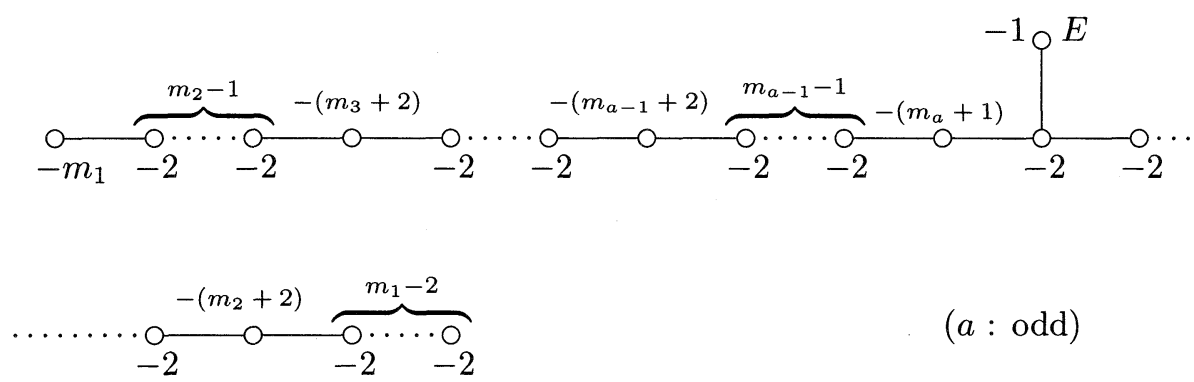

( $a$ : odd)

Figure 1

called a log del Pezzo surface of rank one, for short) (see [11] for the definition).

In the case $(\mathrm{A})$, such pairs $(X, D)$ are completely classified as follows.

Theorem 0.1 (cf. [4, Theorem 1.1]). Let the notation and the assumptions be the same as above. Then the following assertions hold:

(1) There exists a $\mathbf{P}^{1}$-fibration $h: X \rightarrow C$ over a curve $C$ such that $\operatorname{Supp}(D)$ is contained in $\operatorname{Supp}\left(F_{0}\right)$, where $F_{0}$ is a unique reducible fiber of $h$. Furthermore, there exists a unique $(-1)$-curve $E$ on $X$ such that $\operatorname{Supp}(E+D)$ coincides with $\operatorname{Supp}\left(F_{0}\right)$.

(2) The weighted dual graph of $E+D$ is one of the following:

(i) Case when $\operatorname{Supp} D$ is a linear chain. Then the dual graph of $E+D$ is given as in Figure 1, where $m_{1} \geq 2, a \geq 2$ and $m_{i} \geq 1$ for $2 \leq i \leq a$.

(ii) Case when $\operatorname{Supp} D$ is not a linear chain. Then the dual graph of $E+D$ is given as in Figures 2 and 3, where $n \geq 0$, and the subgraph denoted by the encircled 
$S_{a}(a \geq 0)$ in Figure 2 stands for one of the linear chains plus the (-1)-curve $E$ given as in Figure 4 , where $m_{1} \geq 3$ and $m_{i} \geq 1$ for $2 \leq i \leq a$.

(Type D)

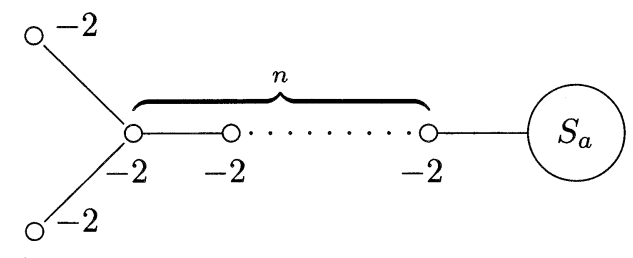

Figure 2

(Type $E_{6}$ )

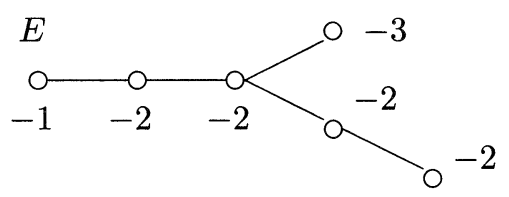

Figure 3

In the case (B), if $P$ is a rational double or triple singular point, then such pairs have been classified completely (see [9] and [12]). But for arbitrary quotient singularities, such pairs are not yet classified.

In the present article, we attempt to classify all the log del Pezzo surfaces of rank one with unique quotient singular points. Our main result is the following:

MaIn Theorem. Let $\bar{X}$ be a log del Pezzo surface of rank one with a unique singular point, say $P$, and let $(X, D)$ be the minimal resolution of $\bar{X}$. Then the weighted dual graph or the configuration of $D$ are listed in Appendices A, B and $\mathrm{C}$. Furthermore, all the listed cases are realizable.

We prove Main Theorem by using Zhang's results on log del Pezzo surfaces of rank one (cf. [11] and [12]) and analyzing singular fibers of $\mathbf{P}^{1}$-fibrations. First of all, we prove in Section 1 certain results on $\log$ del Pezzo surfaces and $\mathbf{P}^{1}$-fibrations. In Section 2, we classify the pairs $(X, D)$ when $P$ is a quotient singular point of type $E_{6}, E_{7}$ or $E_{8}$ (Theorem 2.1). In Section 3, we classify the pairs $(X, D)$ when $P$ is a quotient singular point of type $D$ (Theorem 3.1). Finally, in Section 4 , the case where $P$ is a cyclic quotient singular point is treated (Theorem 4.1). 


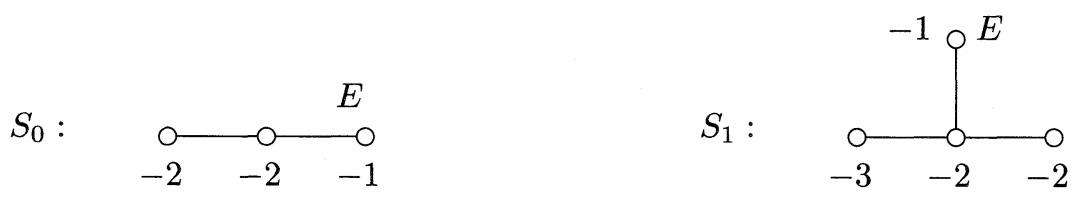

$S_{a}(a \geq 2):$
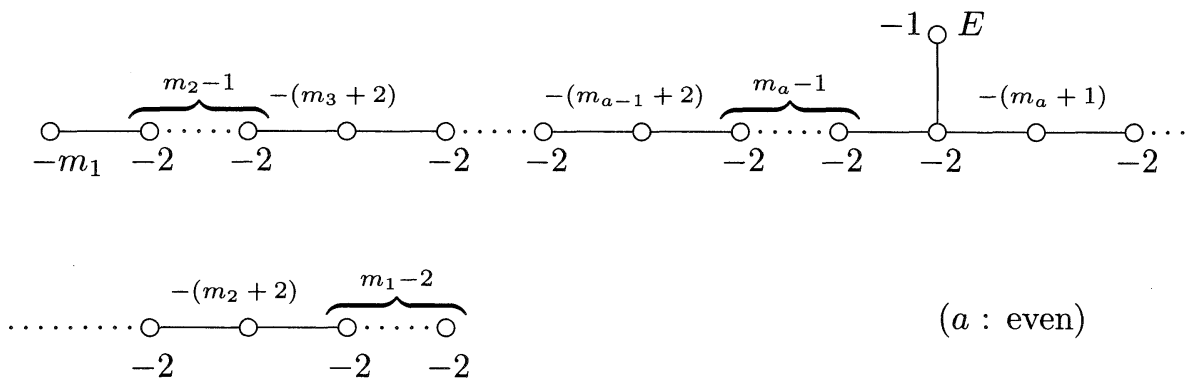

(a: even)
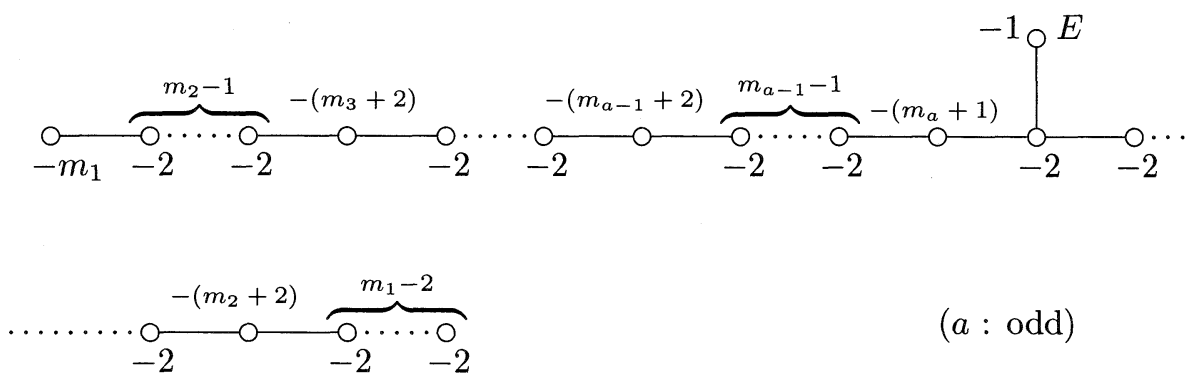

$$
(a \text { : odd })
$$

Figure 4

TERMINOLOGY. A $(-n)$-curve is a nonsingular rational curve with self intersection number $-n$. For the definitions of rods, twigs and forks, we refer to [7]. A reduced effective divisor $D$ is called an NC (resp. SNC) divisor if $D$ has only normal (resp. simple normal) crossings. We employ the following notation:

$K_{X}$ (or simply, $K$ ) : the canonical divisor on $X$.

$\bar{\kappa}(X-D)$ : the logarithmic Kodaira dimension of an open surface $X-D$.

$\rho(X)$ : the Picard number of $X$.

$\mathbf{F}_{n}(n \geq 0)$ : a Hirzebruch surface of degree $n$.

$M_{n}(n \geq 0)$ : the minimal section of $\mathbf{F}_{n}$.

$D^{\#}:=D-\mathrm{Bk}(D)$.

$\# D$ : the number of all irreducible components in $\operatorname{Supp}(D)$. 
The author expresses his gratitude to Professor M. Miyanishi who gave the author valuable advice and constant encouragement during the preparation of the present article. The author also thank the referee for suggestions which make the paper more readable.

\section{Preliminary results}

In this section, we shall state some results in [11] and [12] and prove some results which will be used in the next sections.

Let $(X, D)$ be the minimal resolution of a log del Pezzo surface $\bar{X}$ of rank one with a unique singular point $P$. Then $\rho(X)=\# D+1$. We have the following:

Lemma 1.1 (cf. [12, Lemma 1.1]). Let $(X, D)$ be as above. Then we have:

(1) $f^{*}\left(K_{\bar{X}}\right) \equiv D^{\#}+K_{X}$ and $-\left(D^{\#}+K_{X}\right)$ is nef and big. Moreover, for any irreducible curve $F,-\left(D^{\#}+K_{X} \cdot F\right)=0$ if and only if $F$ is a component of $D$.

(2) Any $(-n)$-curve with $n \geq 2$ is a component of $D$.

(3) $X$ is a rational surface.

Lemma 1.2 (cf. [11, Lemma 1.4]). There is no (-1)-curve E such that, after contracting $E$ and consecutively (smoothly) contractible curves in $E+D$, the divisor $E+D$ becomes an admissible rational rod or fork, where the adjective "admissible" means that each irreducible component of the image of $E+D$ has self intersection number $\leq-2$.

By Lemma $1.1(1)$, one can find an irreducible curve $C$ such that $-\left(C \cdot D^{\#}+\right.$ $\left.K_{X}\right)$ attains the smallest positive value.

Lemma 1.3. Suppose that $\left|C+D+K_{X}\right| \neq \emptyset$ and $P$ is not a rational double point. Then $D$ is an admissible rod, i.e., $P$ is a cyclic quotient singular point.

Proof. By [11, Lemma 2.1], there exists a unique decomposition of $D$ as a sum of effective integral divisors $D=D^{\prime}+D^{\prime \prime}$ such that:

(i) $\left(C \cdot D_{i}\right)=\left(D^{\prime \prime} \cdot D_{i}\right)=\left(K_{X} \cdot D_{i}\right)=0$ for any component $D_{i}$ of $D^{\prime}$.

(ii) $C+D^{\prime \prime}+K_{X} \sim 0$.

If $D^{\prime \prime}=0$ then $D=D^{\prime}$, where $D^{\prime}$ consists of $(-2)$-curves. This contradicts the hypothesis. Hence, $D^{\prime \prime} \neq 0$ and $D=D^{\prime \prime}$ because $\operatorname{Supp}(D)$ is connected. By $[12$, Lemma $1.7(2)] D$ is an admissible rod.

Q.E.D.

Suppose that $\left|C+D+K_{X}\right| \neq \emptyset$ and $D^{\prime \prime}=0$ in the proof of Lemma 1.3. Such a pair $(X, D)$ is then completely determined. Namely, we have the following result.

Lemma 1.4 (cf. [9, Lemma 3] and [12, Lemma 5.1]). With the notation as above, suppose that $\left|C+D+K_{X}\right| \neq \emptyset$ and $D^{\prime \prime}=0$. Then $P$ is a rational double 
point and the dual graph of $D$ is one of the following Dynkin graphs:

$$
A_{1}, A_{4}, D_{5}, E_{6}, A_{7}, E_{7}, D_{8}, E_{8}, A_{8} \text {. }
$$

Moreover, the above $(X, D)$ is obtained from the Hirzebruch surface $\mathbf{F}_{2}$ of degree 2 by a sequence of blowing-ups. The configuration or the weighted dual graph of $D$ is given as one of the cases in Appendices A, B and C.

Suppose that $\left|C+D+K_{X}\right|=\emptyset$. The divisor $C+D$ is then an SNC-divisor, consisting of nonsingular rational curves and the dual graph of $C+D$ is a tree (cf. [6, Lemma 2.1.3]). Since $\operatorname{Supp}(D)$ is connected, $C \cap D=\emptyset$ or $C$ meets only one irreducible component of $D$ transversally. More precisely, we have the following:

Lemma 1.5 (cf. [11, Lemma 2.2] and [2, Proposition 3.6]). Suppose that $\mid C+$ $D+K_{X} \mid=\emptyset$. Then $(X, D)$ is $\left(\mathbf{F}_{n}, M_{n}\right)$, where $n=-\left(D^{2}\right) \geq 2$, or we may assume that $C$ is a (-1)-curve.

Proposition 1.6. Suppose that $\left|C+D+K_{X}\right|=\emptyset$ and $D$ is an admissible rational fork. Let $D_{0}$ be the unique irreducible component of $D$ such that $\left(D_{0} \cdot D-\right.$ $\left.D_{0}\right)=3$. Then $C$ does not meet $D_{0}$.

Proof. Since $D$ is a fork, $C$ is a $(-1)$-curve by Lemma 1.5. Suppose that $\left(C \cdot D_{0}\right)=1$. Then $D_{0}$ is a $(-2)$-curve by Lemma 1.2 . Let $\mu: X \rightarrow \tilde{X}$ be the contraction of $C$ and let $\tilde{D}=\mu(D)$. By an argument similar to [11, Lemma 6.4], we know that $(\tilde{X}, \tilde{D})$ is a log del Pezzo surface of rank one with non-contractible boundary (for the definition, see Miyanishi-Tsunoda [8]). By Lemma 2.6 and Theorems 4 and 6 in [8], $\tilde{D}$ consists of a fork and an admissible rational fork which are disjoint from each other. This is however a contradiction because $\operatorname{Supp}(D)$ (and hence $\operatorname{Supp}(\tilde{D}))$ is connected.

Q.E.D.

In the rest of this section we prove some lemmas concerning $\mathbf{P}^{1}$-fibrations which we need later.

Lemma 1.7 (cf. [12, Lemma 1.5(1)]). Let $\Phi: X \rightarrow \mathbf{P}^{1}$ be a $\mathbf{P}^{1}$-fibration on a nonsingular projective rational surface $X$. Suppose that there are two crosssections $H_{1}$ and $H_{2}$ of $\Phi$ such that $\left(H_{i}^{2}\right) \leq-2$ for $i=1,2$. Let $\mu: X \rightarrow \mathbf{F}_{m}$ be the contraction of all $(-1)$-curves and consecutively contractible curves in singular fibers so that $\left(\mu_{*}\left(H_{1}\right)^{2}\right)=-m=\left(H_{1}^{2}\right)$. Then

$$
\left(\mu_{*}\left(H_{2}\right)^{2}\right)=m+2\left(H_{1} \cdot H_{2}\right) .
$$

Proof. By the assumption on $\mu$, it follows that $H_{1}$ meets none of the curves contracted by $\mu$. Hence we have

$$
\left(\mu_{*}\left(H_{1}\right) \cdot \mu_{*}\left(H_{2}\right)\right)=\left(H_{1} \cdot H_{2}\right)
$$


and

$$
\mu_{*}\left(H_{2}\right) \sim \mu_{*}\left(H_{1}\right)+\left(m+\left(H_{1} \cdot H_{2}\right)\right) \ell
$$

where $\ell$ is a general fiber of the $\mathbf{P}^{1}$-fibration $\Phi \circ \mu^{-1}: \mathbf{F}_{m} \rightarrow \mathbf{P}^{1}$. Hence

$$
\left(\mu_{*}\left(H_{2}\right)^{2}\right)=\left(\mu_{*}\left(H_{1}\right)^{2}\right)+2 m+2\left(H_{1} \cdot H_{2}\right)=m+2\left(H_{1} \cdot H_{2}\right) .
$$

Q.E.D.

Lemma 1.8. Let $\mu: \mathbf{F}_{n} \rightarrow \mathbf{P}^{1}(n \geq 0)$ be the ruling on $\mathbf{F}_{n}$ and let $M$ be an $m$-section $(m \geq 1)$ of $\mu$ different from $M_{n}$. Then $\left(M^{2}\right) \geq n m^{2}$.

Proof. We may assume that $n \geq 1$. Since $M$ is an $m$-section there exists an integer $\alpha$ such that $M \sim m M_{n}+\alpha \ell$, where $\ell$ is a general fiber of the ruling. Note that $\alpha \geq n m$ because $M$ is irreducible and $M \neq M_{n}$. Hence

$$
\left(M^{2}\right)=-n m^{2}+2 \alpha m \geq n m^{2} .
$$

Q.E.D.

\section{The case of type $E_{n}$}

Let $(X, D)$ be the same pair as in Main Theorem. In the present section, we consider the case where $P$ is a quotient singular point of type $E_{6}, E_{7}$ or $E_{8}$. The main result is stated as follows:

Theorem 2.1. Let $\bar{X}$ be a log del Pezzo surface of rank one with a unique singular point $P$ and let $(X, D)$ be the minimal resolution of $\bar{X}$. Suppose that $P$ is a quotient singular point of type $E_{6}, E_{7}$ or $E_{8}$. Then the following assertions hold:

(1) If $P$ is a rational double point, then there exists a $(-1)$-curve $E$ such that the configuration of $E+D$ is one of (2), (4) and (9) in Appendix A.

(2) If $P$ is not a rational double point, then the configuration of $C+D$ with the curve $C$ as in the previous section is one of those except for (2), (4) and (9) in Appendix A.

(3) There exist a $\mathbf{P}^{1}$-fibration $\Phi: X \rightarrow \mathbf{P}^{1}$ and a component $H$ of $D$ such that $H$ is a cross-section of $\Phi$ and the other components of $E+D$ (if $P$ is a rational double point) or $C+D$ (if $P$ is not a rational double point) are contained in a unique singular fiber of $\Phi$.

(4) All the cases listed in Appendix A are realizable.

Proof. The case where $P$ is a rational double point follows from $[9$, Lemma $3]$. Hence we treat the case where $P$ is not a rational double point. 
There are 15 types of Dynkin graphs for the quotient singularities of types $E_{6}$, $E_{7}$ and $E_{8}$ (cf. [1], [5]). Our proof is to verify the assertions of the above theorem by checking these cases separately. Since the reasoning is more or less the same for all cases, we exhibit the idea of the proof in the case where the configuration of $D$ is given as in Figure 5 , where $D_{5}$ is a $(-3)$-curve, $D_{0}$ is a $\left(-a_{0}\right)$-curve with $a_{0} \geq 2$ and the other curves are $(-2)$-curves. The type of the quotient singular point $P$ is $E_{8}$.

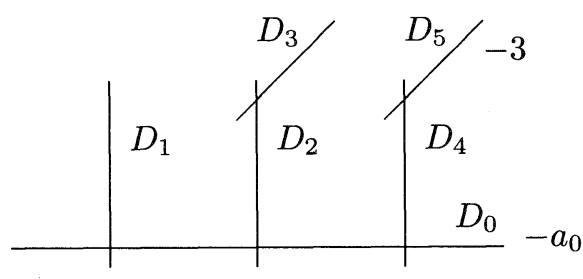

Figure 5

As in the section one, $C$ is an irreducible curve such that $-\left(C \cdot D^{\#}+K\right)$ attains the smallest positive value. Since $P$ is not a rational double point, we may assume, by Lemmas 1.3 and 1.5 , that $|C+D+K|=\emptyset$ and $C$ is a $(-1)$-curve. It follows that $(C \cdot D)=1$. Lemma 1.2 and Proposition 1.6 imply that $C$ meets only one of the $D_{i}(1 \leq i \leq 4)$. We consider the following four cases separately.

Case 1: $\quad\left(C \cdot D_{1}\right)=1$. By Lemma 1.2 and since $a_{0} \geq 2$, we conclude $a_{0}=2$. Hence $P$ is a rational triple singular point. By [12, Main Theorem] this is, however, not the case. For the sake of the reader's convenience, we shall reproduce the proof.

Let $S_{0}=2\left(C+D_{0}+D_{1}\right)+D_{2}+D_{4}$ and let $\Phi: X \rightarrow \mathbf{P}^{1}$ be the $\mathbf{P}^{1}$-fibration defined by the linear pencil $\left|S_{0}\right|$. Then $D_{3}$ and $D_{5}$ are cross-sections of $\Phi$. Since $\rho(X)=\# D+1=7$ and $\# S_{0}-1=4$, there exists another singular fiber $S_{1}=$ $F_{1}+F_{2}$, where $F_{1}$ and $F_{2}$ are $(-1)$-curves. By the count of $\rho(X)$, we know that $\Phi$ has no singular fibers other than $S_{0}$ and $S_{1}$. Since $(X, D)$ is almost minimal, the $(-1)$-curves $F_{1}$ and $F_{2}$ have to meet the cross-sections $D_{3}$ and $D_{5}$. We may assume that $\left(F_{1} \cdot D_{3}\right)=\left(F_{2} \cdot D_{5}\right)=1$. Then $\left(F_{2} \cdot D\right)=\left(F_{2} \cdot D_{5}\right)=1$. Since $\left(D_{5}^{2}\right)=-3$, $F_{2}+D$ can be contracted to a quotient singular point. This contradicts Lemma 1.2. Thus the above case cannot occur.

Case 2: $\left(C \cdot D_{2}\right)=1$. By Lemma $1.2, a_{0}=2$ or 3 . If $a_{0}=2$ then $P$ is a rational triple singular point. By [12, Main Theorem] this is not the case. Hence $a_{0}=3$. Let $S_{0}=4\left(C+D_{2}\right)+2\left(D_{3}+D_{0}\right)+D_{1}+D_{4}$ and let $\Phi: X \rightarrow \mathbf{P}^{1}$ be the $\mathbf{P}^{1}$-fibration defined by the linear pencil $\left|S_{0}\right|$. Then $D_{5}$ is a cross-section of $\Phi$ and the fibration $\Phi$ has no singular fibers other than $S_{0}$, since $\# S_{0}-1=5$ and 
$\rho(X)=7$. The configuration of $C+D$ is then given as (7) in Appendix A.

Case 3: $\quad\left(C \cdot D_{3}\right)=1$. By Lemma 1.2, $a_{0}=2$. Let $S_{0}=2\left(C+D_{0}+D_{2}+\right.$ $\left.D_{3}\right)+D_{1}+D_{4}$ and let $\Phi: X \rightarrow \mathbf{P}^{1}$ be the $\mathbf{P}^{1}$-fibration defined by the linear pencil $\left|S_{0}\right|$. Then $D_{5}$ is a cross-section of $\Phi$ and $S_{0}$ is a unique singular fiber of $\Phi$. The configuration of $C+D$ is then given as (8) in Appendix A (see also Appendix in [12]).

Case 4: $\quad\left(C \cdot D_{4}\right)=1$. By Lemma $1.2, a_{0}=2$. Then $P$ is a rational triple singular point, and this is not the case by [12, Main theorem].

The above argument shows that if $D$ is as in Figure 5 then $C+D$ has the configuration (7) or (8) in Appendix A. We can construct the configurations (7) and (8) starting from the Hirzebruch surface $\mathbf{F}_{3}$ of degree 3.

\section{The case of type $D$}

Let $(X, D)$ be an almost minimal pair as in Main Theorem. In this section we assume that the quotient singular point $P$ has type $D$. The weighted dual graph of $D$ is given as in Figure 6, where $a_{i} \geq 2$ for $i=0,3, \ldots, r$.

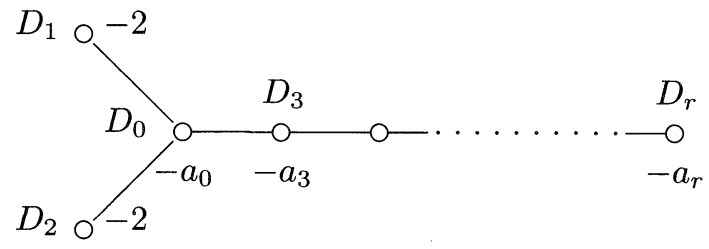

Figure 6

Note that $\rho(X)=r+2$ and $\# D=r+1$. Our main result of this section is the following:

Theorem 3.1. Let $\bar{X}$ be a log del Pezzo surface of rank one with a unique singular point $P$ and let $(X, D)$ be the minimal resolution of $\bar{X}$. Suppose that $P$ is a quotient singular point of type $D$ and that the weighted dual graph of $D$ is given as in Figure 6. Then we have the following:

(1) There exists a $(-1)$-curve $E$ such that $(E \cdot D)=\left(E \cdot D_{t}\right)=1$, where $t=1$ or 2. The weighted dual graph of $E+D$ is given as in Appendix $\mathrm{B}$.

(2) There exists a $\mathbf{P}^{1}$-fibration $\Phi: X \rightarrow \mathbf{P}^{1}$ such that $D_{4}$ is a cross-section of $\Phi$ and the other components of $D$ are contained in at most two singular fibers of $\Phi$. In particular, the $D_{i}(0 \leq i \leq 3)$ are $(-2)$-curves.

(3) All of the types of singularities listed in Appendix B are realizable. 
In what follows, we shall prove the above theorem. The case where $P$ is a rational double point follows from [9, Lemma 3]. Hence we treat the case where $P$ is not a rational double point. Let $C$ be an irreducible curve on $X$ such that $-\left(C \cdot D^{\#}+K\right)$ attains the smallest positive value. By Lemmas 1.3 and 1.5 , we may assume that $C$ is a $(-1)$-curve and that $\left|C+D+K_{X}\right|=\emptyset$. Then $C$ meets $D$ only in one component, say $D_{s}(0 \leq s \leq r)$. By Proposition 1.6, $s \neq 0$, i.e., $s \geq 1$.

First, we consider the case $s=1$ (similarly, $s=2$ ). We then have the following:

Lemma 3.2. Suppose that $s=1$. Then $r \geq 4, a_{0}=a_{3}=2$ and $S_{0}:=$ $2\left(C+D_{0}+D_{1}\right)+D_{2}+D_{3}$ defines a $\mathbf{P}^{1}$-fibration $\Phi: X \rightarrow \mathbf{P}^{1}$ such that $\Phi$ has at most two singular fibers. The weighted dual graph of $C+D$ is given as in Appendix $\mathrm{B}$, where $E$ is to be read as $C$.

Proof. By Lemma 1.2, $a_{0}=2$. Similarly, $a_{3}=2$. Hence $S_{0}:=2\left(C+D_{0}+\right.$ $\left.D_{1}\right)+D_{2}+D_{3}$ gives rise to a $\mathbf{P}^{1}$-fibration $\Phi: X \rightarrow \mathbf{P}^{1}$. Since $\rho(X)=r+2$ we know that $r \geq 4$ and $D_{4}$ is a cross-section of $\Phi$.

Suppose that $r \geq 5$. Let $S_{1}$ be the singular fiber of $\Phi$ containing $D_{i}(5 \leq i \leq r)$. Then,

$$
\rho(X)=r+2 \geq 2+\left(\# S_{0}-1\right)+\left(\# S_{1}-1\right)=\# S_{1}+5 .
$$

Further, \# $S_{1} \geq r-3$ because $S_{1}$ contains $D_{5}, \ldots, D_{r}$ and some $(-1)$-curves. Hence there exists a unique $(-1)$-curve $E_{1}$ in $\operatorname{Supp}\left(S_{1}\right)$ and $\Phi$ has no singular fibers other than $S_{0}$ and $S_{1}$. Put $E:=C$. The weighted dual graph of $E+D$ is then given as in Appendix B.

Q.E.D.

By Lemma 3.2, we may assume, in the subsequent arguments, that $s \geq 3$. Since $\left(C \cdot D^{\#}+K_{X}\right)<0$ and $(X, D)$ is almost minimal, the intersection matrix of $C+D$ is not negative definite. Furthermore, since $\rho(X)=\# D+1=r+2$, the intersection matrix of $C+D$ is not negative semi-definite, either. Hence there exist an integer $e>0$ and an effective divisor $\Delta_{0}$ with $\operatorname{Supp}\left(\Delta_{0}\right) \subset \operatorname{Supp}(D)$ such that $\left|e C+\Delta_{0}\right|$ defines a $\mathbf{P}^{1}$-fibration $\Phi: X \rightarrow \mathbf{P}^{1}$. Put $S_{0}=e C+\Delta_{0}$ and put $\Delta=\left(\Delta_{0}\right)_{\text {red }}$. Hereafter in the present section, we retain this situation.

Lemma 3.3. Suppose that $\Delta$ is an admissible rational fork, i.e., $\Delta=D_{0}+$ $D_{1}+D_{2}+D_{3}+\cdots+D_{i}$ for some $3 \leq i \leq r$. Then we have:

(1) $i=r-1$ and $D_{r}$ is a 2-section of $\Phi$. Furthermore, $\Phi$ has no singular fibers other than $S_{0}$.

(2) There exists a $(-1)$-curve $E$ such that $(E \cdot D)=\left(E \cdot D_{t}\right)=1$, where $t=1$ or 2 . Hence the weighted dual graph of $E+D$ is given as in Appendix B.

Proof. (1) Since $\rho(X)=r+2$, it follows that $i<r$. The weighted dual graph of $C+\Delta$ looks like that of Figure 2, where the subgraph denoted by the 
encircled $S_{a}(a \geq 0)$ is given as in Figure 4. As seen from Figure 2 the multiplicity of $D_{i}$ in $S_{0}$ is equal to two. Hence $D_{i+1}$ is a 2 -section of $\Phi$. Suppose that $i \leq r-2$. Let $S_{1}$ be a singular fiber of $\Phi$ containing $D_{i+2}, \ldots, D_{r}$. By the count of $\rho(X)$, we know that $S_{1}$ contains a unique (-1)-curve $E_{1}$ and $\Phi$ has no singular fibers other than $S_{0}$ and $S_{1}$. The weighted dual graph of $\operatorname{Supp}\left(S_{1}\right)$ is then the one in Figure 1. Since $D_{i+2}$ and $D_{r}$ are terminal components of $\operatorname{Supp}\left(S_{1}\right)$, the multiplicity of $D_{i+2}$ in $S_{1}$ is equal to one. Then $\left(E_{1} \cdot D_{i+1}\right)=1$. Since the multiplicity of $E_{1}$ in $S_{1}$ is then equal to one, there exists another $(-1)$-curve in $S_{1}$. This is a contradiction. Hence $i=r-1$. By the count of $\rho(X), \Phi$ then has no singular fibers other than $S_{0}$.

(2) Let $f: X \rightarrow \mathbf{F}_{m}$ be a sequence of contractions of (-1)-curves in a unique singular fiber $S_{0}$ of $\Phi$. Then $m=0$ or 1 . Indeed, if $m \geq 2$ then the proper transform $f^{\prime}\left(M_{m}\right)$ of $M_{m}$ is a nonsingular rational curve with $\left(f^{\prime}\left(M_{m}\right)^{2}\right) \leq-2$ and $f^{\prime}\left(M_{m}\right)$ is not contained in $\operatorname{Supp}(D)$. This contradicts Lemma $1.1(2)$.

In order to prove the assertion, we consider two cases $m=1$ and $m=0$ separately.

Case: $m=1$. Put $\ell=f_{*}\left(S_{0}\right)$. Then there exists a unique fundamental point $Q$ of $f$ on $\ell$. Let $E^{\prime}$ be the proper transform of $M_{1}$ on $X$. Since $f_{*}\left(D_{r}\right) \sim$ $2 M_{1}+\alpha \ell$ with $\alpha \geq 2$ we have $\left(f_{*}\left(D_{r}\right)^{2}\right)=-4+4 \alpha \geq 4$. Furthermore, by the above contractions, $f_{*}\left(D_{r}\right)$ remains as a nonsingular rational curve. Since

$$
-2=\left(f_{*}\left(D_{r}\right)^{2}\right)+\left(f_{*}\left(D_{r}\right) \cdot K_{\mathbf{F}_{m}}\right)=2(\alpha-3),
$$

we have $\alpha=2$ and $\left(f_{*}\left(D_{r}\right) \cdot M_{1}\right)=0$. In particular, $Q \notin M_{1}$. Hence we know that $E^{\prime}$ is a $(-1)$-curve with $\left(E^{\prime} \cdot D\right)=\left(E^{\prime} \cdot D_{t}\right)=1$, where $t=1$ or 2 .

Case: $m=0$. Let $\ell$ and $Q$ be the same as in the case $m=1$. Put $\bar{D}_{r}:=$ $f_{*}\left(D_{r}\right)$. Since $\bar{D}_{r} \sim 2 M_{0}+\alpha \ell$ with $\alpha>0$, we have $\left(\bar{D}_{r} \cdot M_{0}\right)=\alpha>0$. Since $\overline{D_{r}}$ is a nonsingular rational curve and since

$$
-2=\left(\bar{D}_{r} \cdot \bar{D}_{r}+K_{\mathbf{F}_{0}}\right)=2(\alpha-2)
$$

we have $\alpha=1$. Let $\ell^{\prime}$ be a fiber of the second $\mathbf{P}^{1}$-fibration on $\mathbf{F}_{0}=\mathbf{P}^{1} \times \mathbf{P}^{1}$ through $Q$. The proper transform $f^{\prime}\left(\ell^{\prime}\right)$ of $\ell^{\prime}$ is then a $(-1)$-curve satisfying

$$
\left(f^{\prime}\left(\ell^{\prime}\right) \cdot D\right)=\left(f^{\prime}\left(\ell^{\prime}\right) \cdot D_{t}\right)=1
$$

where $t=1$ or 2 .

This completes the proof of Lemma 3.3.

In view of Lemma 3.3, we next consider the case where $\Delta$ is a linear chain. The weighted dual graph of $C+\Delta$ is given as in Figure 1, where $E$ is to be read as $C$. 
Proposition 3.4. With the notation and assumptions as above, let $D_{i_{0}}$ and $D_{i_{1}}$ be the terminal components of the linear chain $\Delta$, where $i_{0}<i_{1}$. Then we have:

(1) $i_{0}=5$ and $i_{1}=r$.

(2) There exists a $(-1)$-curve $E(\neq C)$ such that $(E \cdot D)=\left(E \cdot D_{t}\right)=1$, where $t=1$ or 2 . The weighted dual graph of $E+D$ is given as in Appendix B.

The proof of Proposition 3.4 consists of Lemmas $3.5 \sim 3.9$.

Lemma 3.5. With the notation as in Proposition 3.4 , we have $i_{0} \geq 3$. Namely, $\Delta$ does not contain $D_{0}$.

Proof. Suppose to the contrary that $\Delta$ contains $D_{0}$. We may assume that $\Delta$ does not contain $D_{2}$. Indeed, suppose that $\Delta$ contains $D_{1}$ and $D_{2}$. Since $\Delta$ is a linear chain, then $\Delta=D_{0}+D_{1}+D_{2}$ and $(C \cdot D)=(C \cdot \Delta)=\left(C \cdot D_{0}\right)=1$. This contradicts Proposition 1.6. Our proof differs depending on whether $\Delta$ contains $D_{1}$ or not.

(I) Suppose that $\Delta$ contains $D_{1}$. The dual graph of $\Delta$ looks like that of Figure 1 and the multiplicity $m$ of $D_{0}$ in $S_{0}$ is larger than 1 . Then $D_{2}$ is an $m$ section $(m \geq 2)$ of $\Phi$. Furthermore, $m=2$. Indeed, in the course of contracting $C$ and successively contractible curves in the fiber $S_{0}=e C+\Delta_{0}$, when the image of $D_{0}$ becomes a $(-1)$-curve, the fiber consists of one (-1)-curve and two ( -2$)$-curves, one of which is the image of $D_{1}$. Hence the multiplicity of the $(-1)$-curve, which is equal to $m$, must be 2 . We consider the following three cases separately.

Case $(\mathrm{I}-1): i_{1}=r$. Since $\rho(X)=r+2$ and $\# S_{0}=r+1$, the $\mathbf{P}^{1}$-fibration $\Phi$ has no singular fibers other than $S_{0}$. Let $f: X \rightarrow \mathbf{F}_{n}$ be a sequence of contractions of $(-1)$-curves and subsequently contractible curves in $\operatorname{Supp}\left(S_{0}\right)$. We call such a sequence of contractions a successive contraction of $(-1)$-curves. We may and shall assume that $f_{*}\left(S_{0}\right)=f_{*}\left(D_{1}\right)$, since the multiplicity of $D_{1}$ in $S_{0}$ is equal to one. By Lemma $1.1(2), n \leq 1$. Put $\bar{D}_{2}=f_{*}\left(D_{2}\right)$. By the construction of $f, \bar{D}_{2}$ is a nonsingular rational curve with $\left(\bar{D}_{2}^{2}\right)=0$. Hence $n=0$ by Lemma 1.8 .

Write $\bar{D}_{2} \sim 2 M_{0}+\alpha \ell$ with $\alpha \geq 0$. Since $\left(\bar{D}_{2}^{2}\right)=0$, it follows that $\alpha=0$. Hence $\bar{D}_{2} \sim 2 M_{0}$. This contradicts the irreducibility of the divisor $\bar{D}_{2}$.

Case (I-2): $\quad i_{1}=r-1$. In this case, $D_{r}$ is a cross-section of $\Phi$. Since $\rho(X)=$ $r+2$ and $\# S_{0}=r, \Phi$ has another singular fiber $S_{1}$ which consists of two $(-1)$ curves, say $E_{0}$ and $E_{1}$, and has no singular fibers other than $S_{0}$ and $S_{1}$. We may assume that $\left(E_{0} \cdot D_{r}\right)=1$.

Claim 1. $\left(E_{0} \cdot D_{2}\right)=\left(E_{1} \cdot D_{2}\right)=1$.

Proof. We have $\left(E_{0} \cdot D_{2}\right)=1$ or 0 . Indeed, if $\left(E_{0} \cdot D_{2}\right)=2$ then $\left(E_{1} \cdot D\right)=0$. This contradicts Lemma 1.2. Suppose that $\left(E_{0} \cdot D_{2}\right)=0$. Then $\left(E_{1} \cdot D_{2}\right)=2$ because 
$S_{1}=E_{0}+E_{1}$ and $D_{2}$ is a 2-section of $\Phi$. Let $g: X \rightarrow \mathbf{F}_{n}$ be a successive contractions of $(-1)$-curves in $S_{0}$ and $S_{1}$ such that $g_{*}\left(S_{0}\right)=g_{*}\left(D_{r-1}\right)$ and $g_{*}\left(S_{1}\right)=g_{*}\left(E_{1}\right)$. Then,

$$
n=-\left(D_{r}^{2}\right)-1=a_{r}-1 \geq 1 \text {. }
$$

Put $\bar{D}_{2}:=g_{*}\left(D_{2}\right)$. Then $\bar{D}_{2}$ is a nonsingular rational curve with $\left(\bar{D}_{2}^{2}\right)=0$. This contradicts Lemma 1.8. Hence $\left(E_{0} \cdot D_{2}\right)=\left(E_{1} \cdot D_{2}\right)=1$.

Let $f: X \rightarrow \mathbf{F}_{n}$ be a successive contractions of $(-1)$-curves in $S_{0}$ and $S_{1}$ such that $f_{*}\left(S_{0}\right)=f_{*}\left(D_{r-1}\right)$ and $f_{*}\left(S_{1}\right)=f_{*}\left(E_{0}\right)$. Then $\left(f_{*}\left(D_{2}\right)^{2}\right)=1$. This also contradicts Lemma 1.8 because $n=-\left(D_{r}^{2}\right)=-a_{r} \geq 2$.

Case (I-3): $i_{1} \leq r-2 . \quad D_{i_{1}+1}$ is a cross-section of $\Phi$. Let $S_{1}$ be a singular fiber of $\Phi$ which contains $D_{i_{1}+2}, \ldots, D_{r}$. Now we prove the following assertion.

Claim 2. $S_{1}$ contains two (-1)-curves, say $E_{0}$ and $E_{1}$.

Proof. $\quad S_{1}$ contains at most two $(-1)$-curves because $\rho(X)=r+2, \# S_{0}=$ $i_{1}+1$ and $\# S_{1} \geq r-i_{1}$. Suppose that $S_{1}$ contains only one $(-1)$-curve, say $E$. The weighted dual graph of $\operatorname{Supp}\left(S_{1}\right)$ then looks like that of Figure 1. By the count of $\rho(X)$, there exists the third singular fiber $S_{2}=E_{0}+E_{1}$, where $E_{0}$ and $E_{1}$ are $(-1)$-curves. Note that $S_{0}, S_{1}$ and $S_{2}$ exhaust all singular fibers of $\Phi$. Since $\left(D_{2} \cdot S_{0}\right)=2$, the multiplicity of $E$ in $S_{1}$ is accordingly equal to 2 . For, if the multiplicity is 1 there should exist another $(-1)$-curve in $S_{1}$. This implies that $i_{1}=r-4, S_{1}=2\left(E+D_{r-1}\right)+D_{r-2}+D_{r}$ and $a_{r-2}=a_{r-1}=a_{r}=2$ (cf. Figure $1)$.

Now we may assume that $\left(E_{0} \cdot D_{r-3}\right)=1$. Then $\left(E_{0} \cdot D_{2}\right) \leq 1$. Indeed, if $\left(E_{0} \cdot D_{2}\right)=2$ then $\left(E_{1} \cdot D\right)=0$, a contradiction. Let $f: X \rightarrow \mathbf{F}_{n}$ be a successive contraction of $(-1)$-curves in $S_{0}, S_{1}$ and $S_{2}$ such that $f_{*}\left(S_{0}\right)=f_{*}\left(D_{r-4}\right), f_{*}\left(S_{1}\right)=$ $f_{*}\left(D_{r-2}\right)$ and $f_{*}\left(S_{2}\right)=f_{*}\left(E_{1}\right)$. Then $n=-\left(D_{r-3}^{2}\right)-1 \geq 1$ and $M_{n}=f_{*}\left(D_{r-3}\right)$. $\bar{D}_{2}=f_{*}\left(D_{2}\right)$ is a nonsingular rational curve and

$$
\left(\bar{D}_{2}^{2}\right)=\left(D_{2}^{2}\right)+2+3+\left\{\left(E_{0} \cdot D_{2}\right)\right\}^{2} \leq 4
$$

Hence, by Lemma 1.8 , we know that $n=1,\left(E_{0} \cdot D_{2}\right)=1$ and $\left(E_{1} \cdot D_{2}\right)=1$. The weighted dual graph of all singular fibers of $\Phi$ and $D_{2}$ and $D_{r-3}$ looks like that of Figure 7.

Let $T_{0}=E+D_{2}+E_{0}$. The complete linear system $\left|T_{0}\right|$ defines a $\mathbf{P}^{1}$-fibration $\Psi: X \rightarrow \mathbf{P}^{1}$ for which $D_{0}, D_{r-3}$ and $D_{r-1}$ are cross-sections and $E_{1}$ is a 2-section. Let $T_{1}$ be a singular fiber containing $D_{3}, \ldots, D_{r-4}$ and $C$ and let $T_{2}$ be a fiber containing $D_{1}$. Then $T_{1}$ (resp. $T_{2}$ ) contains $D_{r}$ (resp. $\left.D_{r-2}\right)$. Indeed, suppose that $T_{1}$ does not contain $D_{r}$. By Lemma $1.1(2)$, there exists a $(-1)$-curve in $\operatorname{Supp}\left(T_{1}\right)$ which meets $D_{r-1}$. Hence $\# T_{1} \geq r-4$. Furthermore, $D_{r}$ and $D_{r-2}$ cannot belong 


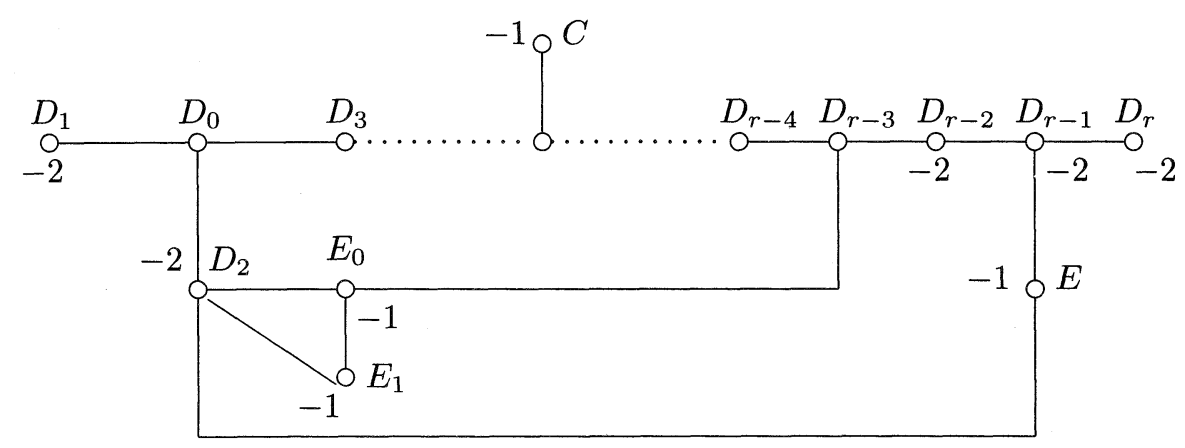

Figure 7

to the same fiber of $\Psi$. This implies that there is the fourth singular fiber $T_{3}$ which contains at least 3 components. Then,

$$
\begin{aligned}
r & \geq\left(\# T_{0}-1\right)+\left(\# T_{1}-1\right)+\left(\# T_{2}-1\right)+\left(\# T_{3}-1\right) \\
& \geq 2+r-5+2+2 \\
& \geq r+1
\end{aligned}
$$

This is a contradiction. Thus $T_{1}$ contains $D_{r}$. Next suppose that $T_{2}$ does not contain $D_{r-2}$. Then $\Psi$ has the fourth singular fiber $T_{3}$ containing $D_{r-2}$. Since $\# T_{2} \geq 3$ and $\# T_{3} \geq 3$, we have

$$
\begin{aligned}
r & \geq\left(\# T_{0}-1\right)+\left(\# T_{1}-1\right)+\left(\# T_{2}-1\right)+\left(\# T_{3}-1\right) \\
& \geq r+2 .
\end{aligned}
$$

This is a contradiction. By the count of $\rho(X)$, all singular fibers of $\Psi$ are exhausted by $T_{0}, T_{1}$ and $T_{2}$. Furthermore, we know that $T_{1}$ has two $(-1)$-curves and $T_{2}$ a unique $(-1)$-curve. Let $\bar{C}$ be a $(-1)$-curve in $\operatorname{Supp}\left(T_{1}\right)$ other than $C$ and let $E_{2}$ be a $(-1)$-curve in $\operatorname{Supp}\left(T_{2}\right)$. Then $T_{2}=D_{1}+2 E_{2}+D_{r-2}$.

We have $\left(\bar{C} \cdot D_{r}\right)=\left(\bar{C} \cdot D_{3}+D_{r-4}\right)=1$. This is because $C+D_{3}+\cdots+D_{r-4}$ (the middle part of the older fiber $S_{0}$ ) is smoothly contractible to a point and the (-1)-curve $\bar{C}$ must link two disjoint parts $C+D_{3}+\cdots+D_{r-4}$ and $D_{r}$ of the fiber $T_{1}$. Suppose that $\left(\bar{C} \cdot D_{r-4}\right)=1$. Then there exists a successive contractions of $(-1)$-curves in $T_{0}, T_{1}$ and $T_{2}$, say $g: X \rightarrow \mathbf{F}_{n}$, such that $g_{*}\left(T_{0}\right)=g_{*}\left(E_{0}\right)$, $g_{*}\left(T_{1}\right)=g_{*}\left(D_{r-4}\right)$ and $g_{*}\left(T_{2}\right)=g_{*}\left(D_{r-2}\right)$. Then $n=-\left(g_{*}\left(D_{r-3}\right)^{2}\right)=-a_{r-3} \geq 2$, $\left(g_{*}\left(D_{r-1}\right)^{2}\right)=-2+3=1$. This contradicts Lemma 1.8. If $\left(\bar{C} \cdot D_{3}\right)=1$ then we have a contradiction similarly.

This completes the proof of Claim 2. 
Let $E_{0}$ and $E_{1}$ be new (-1)-curves contained in $S_{1}$. Then $\Phi$ has exactly two singular fibers $S_{0}$ and $S_{1}$. We may assume that $\left(E_{0} \cdot D_{2}\right)>0$. Since $D_{2}$ is a 2 section of $\Phi$, the multiplicity $m_{0}$ of $E_{0}$ in $S_{1}$ is equal to one or two and $\left(E_{0} \cdot D_{2}\right)=1$ or 2. Now we consider the following three subcases separately:

Subcase (I-3-1): $m_{0}=2$. Then $\left(E_{0} \cdot D_{2}\right)=1$. Let $\mu: X \rightarrow X^{\prime}$ be a successive contraction of $(-1)$-curves in $S_{1}$ such that $\mu_{*}\left(E_{0}\right)$ is a $(-1)$-curve and $\mu_{*}\left(E_{0}\right)$ is a unique $(-1)$-curve of $\mu_{*}\left(S_{1}\right)$. Since $\operatorname{Supp}\left(\left(S_{1}\right)_{\text {red }}-E_{0}-E_{1}\right)$ is a linear chain, the weighted dual graph of $\mu_{*}\left(S_{1}\right)$ looks as in Figure 1 . So, since $m_{0}=2$ one can construct a successive contraction of (-1)-curves in $S_{0}$ and $S_{1}$, say $f: X \rightarrow \mathbf{F}_{n}$, such that $f_{*}\left(D_{2}\right)$ is a nonsingular rational curve with $\left(f_{*}\left(D_{2}\right)^{2}\right)=3$. Hence $n=0$ by Lemma 1.8. Then $\bar{D}_{2}:=f_{*}\left(D_{2}\right) \sim 2 M_{0}+\alpha \ell$ with $\alpha>0$. On the other hand, we have $3=\left(\bar{D}_{2}^{2}\right)=4 \alpha$. This is a contradiction.

Subcase (I-3-2): $m_{0}=1$ and $\left(E_{0} \cdot D_{2}\right)=2$. Let $f: X \rightarrow \mathbf{F}_{n}$ be a successive contraction of $(-1)$-curves in $S_{0}$ and $S_{1}$ such that $f_{*}\left(S_{0}\right)=f_{*}\left(D_{1}\right)$ and $f_{*}\left(S_{1}\right)=$ $f_{*}\left(E_{0}\right)$. Then $\bar{D}_{2}:=f_{*}\left(D_{2}\right)$ is a nonsingular rational curve with $\left(\bar{D}_{2}^{2}\right)=0$. Hence $n=0$ by Lemma 1.8. Then $\bar{D}_{2} \sim 2 M_{0}+\alpha \ell$ with $\alpha \geq 0$. Since $\left(\bar{D}_{2}^{2}\right)=0$, it follows that $\alpha=0$. This is a contradiction.

Subcase (I-3-3): $m_{0}=1$ and $\left(E_{0} \cdot D_{2}\right)=1$. It then follows that $\left(E_{1} \cdot D_{2}\right)=1$ and the multiplicity of $E_{1}$ in $S_{1}$ is equal to one. Since $\operatorname{Supp}\left(S_{1}-E_{0}-E_{1}\right)=$ $D_{i_{1}+2}+\cdots+D_{r}$ is a linear chain, the weighted dual graph of $S_{1}$ is given as in Figure 8, where we assume that $\left(E_{0} \cdot D_{i_{1}+2}\right)=\left(E_{1} \cdot D_{r}\right)=1$.

\begin{tabular}{|c|c|c|}
\hline-1 & $\begin{array}{ll}-2 & -2 \\
-0 & 0 .\end{array}$ & -2 \\
\hline$F$ & $D_{i_{1}+2} D_{i_{1}+3}$ & $D_{r}$ \\
\hline
\end{tabular}

Figure 8

Then $S_{1}=E_{0}+D_{i_{1}+2}+\cdots+D_{r}+E_{1}$. Let $f: X \rightarrow \mathbf{F}_{n}$ be a successive contraction of $(-1)$-curves in $S_{0}$ and $S_{1}$ such that $f_{*}\left(S_{0}\right)=f_{*}\left(D_{i_{1}}\right)$ and $f_{*}\left(S_{1}\right)=f_{*}\left(E_{0}\right)$. Then $n=-\left(D_{i_{1}+1}^{2}\right)-1 \geq 1$ and $f_{*}\left(D_{i_{1}+1}\right)=M_{n}$. Furthermore, $\bar{D}_{2}:=f_{*}\left(D_{2}\right)$ is a nonsingular rational curve with $\left(\bar{D}_{2}^{2}\right)=r-i_{1} \geq 1 . \bar{D}_{2} \sim 2 M_{n}+\alpha \ell$ with $\alpha \geq 2 n$, where $\ell$ is a fiber of the ruling on $\mathbf{F}_{n}$. Then, by the construction of $f,\left(\bar{D}_{2} \cdot M_{n}\right)=1$ and hence $\alpha=2 n+1$. This implies that $p_{a}\left(\bar{D}_{2}\right)=n \geq 1$, where $p_{a}\left(\bar{D}_{2}\right)$ is the arithmetic genus of $\bar{D}_{2}$. This is a contradiction.

(II) We continue the proof of Lemma 3.5. From the arguments in (I), we know that $\Delta$ does not contain $D_{1}$. Since the multiplicity of $D_{0}$ in $S_{0}$ is then equal to one (with the notation before Lemma 3.3), $D_{1}$ and $D_{2}$ are cross-sections of $\Phi$. Put $\Delta=D_{0}+D_{3}+\cdots+D_{i_{1}}\left(i_{1} \geq 4\right)$. As in the case (I), we consider the following 
three cases separately.

Case (II-1): $i_{1}=r$. Since $\rho(X)=r+2$ and $\# S_{0}=r$, there exists a singular fiber $S_{1}=E_{0}+E_{1}$ of $\Phi$, where $E_{0}$ and $E_{1}$ are $(-1)$-curves. Then $\Phi$ has no singular fibers other than $S_{0}$ and $S_{1}$. Assume that $\left(E_{0} \cdot D_{1}\right) \geq 1$. Then, by Lemma 1.2, $\left(E_{0} \cdot D_{1}\right)=\left(E_{1} \cdot D_{2}\right)=1$ and $\left(E_{0} \cdot D_{2}\right)=\left(E_{1} \cdot D_{1}\right)=0$. Let $f: X \rightarrow \mathbf{F}_{n}$ be a successive contraction of $(-1)$-curves in $S_{0}$ and $S_{1}$ such that $f_{*}\left(S_{0}\right)=f_{*}\left(D_{0}\right)$ and $f_{*}\left(S_{1}\right)=f_{*}\left(E_{0}\right)$. Then $f_{*}\left(D_{1}\right)$ and $f_{*}\left(D_{2}\right)$ have negative self-intersection numbers. This is a contradiction.

Case (II-2): $i_{1}=r-1$. Then $D_{r}$ becomes a cross-section of $\Phi$. By Lemma 1.1 (2) and by the count of $\rho(X)$, there exist two other singular fibers $S_{1}=E_{0}+E_{1}$ and $S_{2}=F_{0}+F_{1}$ where $E_{0}, E_{1}, F_{0}$ and $F_{1}$ are $(-1)$-curves. Assume that $\left(E_{0} \cdot D_{1}\right)=$ $\left(F_{0} \cdot D_{1}\right)=1$. Let $f: X \rightarrow \mathbf{F}_{n}$ be a successive contraction of $(-1)$-curves in $S_{0}$, $S_{1}$ and $S_{2}$ such that $f_{*}\left(S_{0}\right)=f_{*}\left(D_{0}\right), f_{*}\left(S_{1}\right)=f_{*}\left(E_{0}\right)$ and $f_{*}\left(S_{2}\right)=f_{*}\left(F_{0}\right)$. Then $n=2$ and $M_{2}=f_{*}\left(D_{1}\right)$. Meanwhile, $\bar{D}_{2}=f_{*}\left(D_{2}\right)$ is a cross-section of the ruling $\Phi \circ f^{-1}: \mathbf{F}_{2} \rightarrow \mathbf{P}^{1}$ with $\left(\bar{D}_{2}^{2}\right) \leq 0$. This contradicts Lemma 1.8 .

Case (II-3): $\quad i_{1} \leq r-2$. Then $D_{i_{1}+1}$ is a cross-section of $\Phi$. Let $S_{1}$ be a singular fiber of $\Phi$ containing $D_{i_{1}+2}, \ldots, D_{r}$. By the count of $\rho(X), S_{1}$ contains at most three $(-1)$-curves. Since $D_{1}$ is a cross-section of $\Phi$, there exists a (-1)-curve, say $E_{1}$, in $S_{1}$ such that $E_{1}$ meets $D_{1}$. Since the multiplicity of $E_{1}$ in $S_{1}$ is then equal to one, $S_{1}$ has two or three $(-1)$-curves. We consider the following two subcases separately:

Subcase (II-3-1). Assume that $S_{1}$ contains three (-1)-curves $E_{1}, E_{2}$ and $E_{3}$ in $S_{1}$. Then $\Phi$ has no other singular fibers. We claim that $\left(E_{1} \cdot D_{2}\right)=0$. Indeed, suppose to the contrary that $\left(E_{1} \cdot D_{2}\right)=1$. Let $f: X \rightarrow \mathbf{F}_{n}$ be a successive contraction of $(-1)$-curves in $S_{0}$ and $S_{1}$ such that $f_{*}\left(S_{0}\right)=f_{*}\left(D_{0}\right)$ and $f_{*}\left(S_{1}\right)=$ $f_{*}\left(E_{1}\right)$. Then $f_{*}\left(D_{1}\right)$ and $f_{*}\left(D_{2}\right)$ are $(-2)$-curves on a Hirzebruch surface. This is a contradiction. We may assume that $\left(E_{2} \cdot D_{2}\right)=1$.

Let $g: X \rightarrow X^{\prime}$ be a successive contraction of $(-1)$-curves in $S_{1}$ such that $S_{1}^{\prime}:=$ $g_{*}\left(S_{1}\right)$ contains only two $(-1)$-curves $g_{*}\left(E_{i}\right)(i=1,2)$. Since $\operatorname{Supp}\left(\left(S_{1}\right)_{\text {red }}-E_{1}-\right.$ $\left.E_{2}-E_{3}\right)$ is a linear chain, the weighted dual graph of $S_{1}^{\prime}$ looks like that of Figure 8. Then $g_{*}\left(D_{i_{1}+1}\right)$ meets a terminal component of $\operatorname{Supp}\left(\left(S_{1}^{\prime}\right)_{\text {red }}-g_{*}\left(E_{1}+E_{2}\right)\right)$, say $G$. We may assume that $\left(g_{*}\left(E_{1}\right) \cdot G\right)=1$. Let $h: X^{\prime} \rightarrow \mathbf{F}_{n}$ be a successive contraction of $(-1)$-curves in $S_{0}^{\prime}\left(:=g_{*}\left(S_{0}\right)\right)$ and $S_{1}^{\prime}$ such that $h_{*}\left(S_{0}^{\prime}\right)=(h \circ g)_{*}\left(D_{0}\right)$ and $h_{*}\left(S_{1}^{\prime}\right)=(h \circ g)_{*}\left(E_{1}\right)$. Put $\bar{D}_{j}:=(h \circ g)_{*}\left(D_{j}\right)\left(j=1,2\right.$ and $\left.i_{1}+1\right)$. Then $\left(\bar{D}_{1}^{2}\right)=-2$ and $\bar{D}_{1}=M_{2}$. Furthermore, $\left(\bar{D}_{1} \cdot \bar{D}_{2}\right)=\left(\bar{D}_{1} \cdot \bar{D}_{i_{1}+1}\right)=0$ and $\left(\bar{D}_{2} \cdot \bar{D}_{i_{1}+1}\right)=1$ by the constructions of $g$ and $h$. Then $\bar{D}_{2} \sim \bar{D}_{i_{1}+1}$ and $\left(\bar{D}_{2}^{2}\right)=2$. This is a contradiction.

Subcase (II-3-2). Assume that $S_{1}$ contains only two (-1)-curves $E_{1}$ and $E_{2}$ and that $\left(E_{1} \cdot D_{1}\right)=1$. Then there exists another singular fiber $S_{2}=F_{1}+F_{2}$, 
where $F_{1}$ and $F_{2}$ are (-1)-curves. By an argument similar to Subcase (II-3-1), it follows that $\left(E_{1} \cdot D_{2}\right)=0,\left(E_{2} \cdot D_{2}\right)=1$ and the weighted dual graph of $S_{1}$ looks like that of Figure 8. Hence we may assume that the configuration of $S_{1}$ is given as in Figure 9 , where $D_{i_{1}+2}, D_{i_{1}+3}, \ldots, D_{r}$ are $(-2)$-curves.

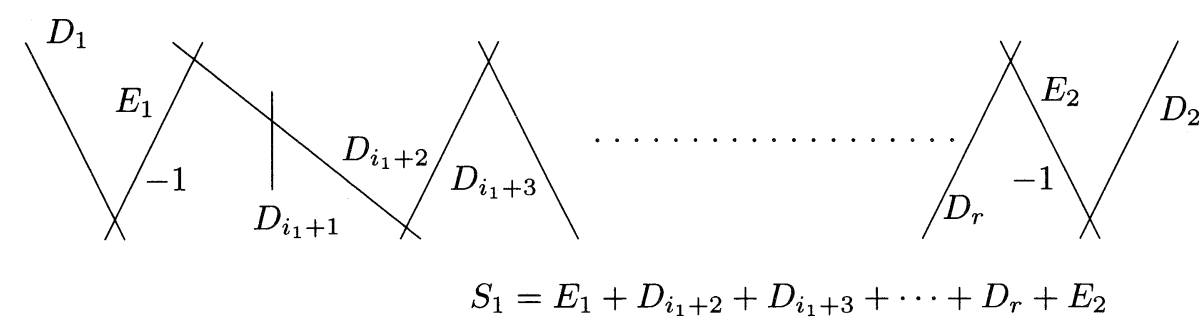

Figure 9

Now assume that $\left(F_{1} \cdot D_{i_{1}+1}\right)=1$. Let $f: X \rightarrow \mathbf{F}_{n}$ be a successive contraction of $(-1)$-curves in $S_{0}, S_{1}$ and $S_{2}$ such that $f_{*}\left(S_{0}\right)=f_{*}\left(D_{0}\right), f_{*}\left(S_{1}\right)=f_{*}\left(E_{1}\right)$ and $f_{*}\left(S_{2}\right)=f_{*}\left(F_{1}\right)$. Put $\bar{D}_{j}=f_{*}\left(D_{j}\right)\left(j=1,2\right.$ and $\left.i_{1}+1\right)$. Since $\left(\bar{D}_{1}^{2}\right) \leq-1$, we have $n=-\left(\bar{D}_{1}^{2}\right) \geq 1$. On the other hand, by the construction of $f,\left(\bar{D}_{i_{1}+1}^{2}\right)=$ $-a_{i_{1}+1}+2 \leq 0$. This contradicts Lemma 1.8 .

This completes the proof of Lemma 3.5.

Lemma 3.6. $\Delta$ does not contain $D_{3}$. Namely, $i_{0} \geq 4$.

Proof. Suppose that $\Delta$ contains $D_{3}$. Put $\Delta=D_{3}+D_{4}+\cdots+D_{i_{1}}$. Then $D_{0}$ and $D_{i+1}$ (if they exist) are cross-sections of $\Phi$. Let $S_{j}$ be a singular fiber of $\Phi$ containing $D_{j}(j=1,2)$. Then $S_{1} \neq S_{2}$ because $D_{0}$ is a cross-section of $\Phi$. Suppose that $i_{1} \geq r-1$. By Lemma $1.1(2), S_{i}(i=1,2)$ consists of one $(-2)$-curve $D_{i}$ and two $(-1)$-curves. Then

$$
\rho(X)=r+2 \geq 2+\left(\# S_{0}-1\right)+\left(\# S_{1}-1\right)+\left(\# S_{2}-1\right) \geq r+3
$$

This is a contradiction. Hence $i_{1} \leq r-2$.

Let $S_{3}$ be a singular fiber of $\Phi$ containing $D_{i_{1}+2}, \ldots, D_{r}$. By the count of $\rho(X), S_{3}$ coincides with either $S_{1}$ or $S_{2}$. Assume that $S_{1}=S_{3}$. By Lemma $1.1(2)$, $S_{2}=F_{1}+D_{2}+F_{2}$, where $F_{1}$ and $F_{2}$ are $(-1)$-curves. By the count of $\rho(X)$, all singular fibers of $\Phi$ are exhausted by $S_{0}, S_{1}$ and $S_{2}$. Furthermore, $S_{1}$ has a unique $(-1)$-curve, say $E_{1}$, and $\left(E_{1} \cdot D_{1}\right)=1$. We claim the following assertion.

Claim. $\left(E_{1} \cdot D_{r}\right)=1$. Furthermore, $i_{1}=r-2$ and $a_{r}=-2$.

Proof. This is clear because the (-2)-curve $D_{1}$ has multiplicity $1 \mathrm{in} S_{1}$. 
Let $f: X \rightarrow \mathbf{F}_{n}$ be a successive contraction of (-1)-curves in $S_{0}, S_{1}$ and $S_{2}$ such that $f_{*}\left(S_{0}\right)=f_{*}\left(D_{3}\right), f_{*}\left(S_{1}\right)=f_{*}\left(D_{1}\right)$ and $f_{*}\left(S_{2}\right)=f_{*}\left(D_{2}\right)$. Then $n=-a_{0} \geq 2$ and $M_{n}=f_{*}\left(D_{0}\right)$. On the other hand,

$$
\left(f_{*}\left(D_{r-1}\right)^{2}\right)=-a_{r-1}+3 \leq 1
$$

because of the construction of $f$. This contradicts Lemma 1.8.

Q.E.D.

We prove the following lemma which will be neccessary later.

Lemma 3.7. Let $F$ be a singular fiber of a $\mathbf{P}^{1}$-fibration $\Phi: X \rightarrow B$. Let $E_{1}, \ldots, E_{s}$ be all $(-1)$-curves in $\operatorname{Supp}(F)$. Suppose that the following conditions are satisfied:

(i) $s \leq 2$.

(ii) $\operatorname{Supp}\left((F)_{\text {red }}-\sum_{i=1}^{s} E_{i}\right)$ consists of two connected components $A$ and $B$.

(iii) $B$ has a component $B_{0}$ whose multiplicity in $F$ is equal to one.

(iv) The dual graph of $A$ is given as in Figure 10, where $\left(A_{1}^{2}\right)=\left(A_{2}^{2}\right)=-2$ and $r \geq 3$. Moreover, the multiplicity of $A_{r}$ in $F$ is equal to one.

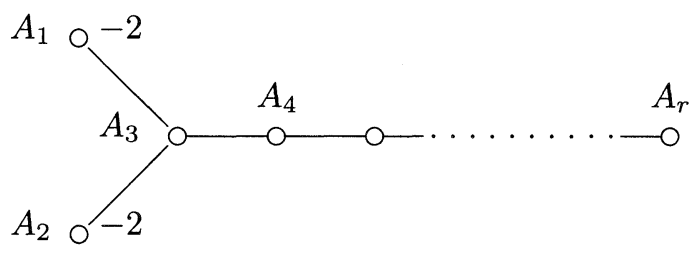

Figure 10

Then we have:

(1) $s=2$.

(2) Suppose that $\left(E_{1} \cdot A\right)=\left(E_{1} \cdot B\right)=1$. Then $\left(E_{2} \cdot A\right)=\left(E_{2} \cdot A_{\ell}\right)$, where $\ell=1$ or 2 .

Proof. Let $E_{1}$ be a $(-1)$-curve in $\operatorname{Supp}(F)$ such that $\left(E_{1} \cdot A\right)=\left(E_{1} \cdot B\right)=1$. Put $T:=A_{3}+\cdots+A_{r}$.

(1) Suppose that $s=1$. Since $\left(E_{1} \cdot A\right)=1$, we may assume that $\left(E_{1} \cdot A_{2}\right)=0$. Let $f: X \rightarrow Y$ be a successive contraction of $(-1)$-curves in $F$ such that $f_{*}\left(A_{3}\right)$ is a $(-1)$-curve. Then $f_{*}\left(A_{2}\right)$ remains as a $(-2)$-curve.

Assume that $r=3$. By the condition (iv), $f_{*}(F)$ has another $(-1)$-curve. In particular, $f_{*}\left(A_{1}+B\right) \neq 0$. Hence $f_{*}\left(A_{3}\right)$ is not a terminal component of $\operatorname{Supp} f_{*}(F)$. This is a contradiction. Assume that $r \geq 4$. By the condition of $f$, we 
know that $f_{*}\left(A_{1}\right) \neq 0$ or $f_{*}\left(A_{4}+\cdots+A_{r}\right) \neq 0$. Since there are no three components meeting a $(-1)$-curve, Supp $f_{*}(F)$ is a linear chain and $f_{*}(B)=0$. On the other hand, by the condition (iii), the multiplicity of $f_{*}\left(A_{3}\right)$ in $f_{*}(F)$ is equal to one. This is a contradiction because $f_{*}\left(A_{3}\right)$ is not a terminal component of $\operatorname{Supp} f_{*}(F)$. Hence we have $s=2$.

(2) Let $E_{2}$ be another $(-1)$-curve in $\operatorname{Supp}(F)$. Then $\left(E_{2} \cdot A\right)=0$ or $\left(E_{2} \cdot B\right)=$ 0 , for otherwise $F$ contains a loop. We prove the following:

Claim. $\left(E_{2} \cdot A\right)=1$.

Proof. Suppose that $\left(E_{2} \cdot B\right)=1$. Let $f^{\prime}: X \rightarrow Y^{\prime}$ be a successive contraction of $(-1)$-curves in $F$ such that $f_{*}^{\prime}\left(E_{1}\right)$ is a $(-1)$-curve and that $f_{*}^{\prime}\left(E_{2}+B\right)$ contains no $(-1)$-curves. By the condition (ii), such a birational morphism $f^{\prime}$ exists. Then Supp $f_{*}^{\prime}(F)$ contains a unique $(-1)$-curve $f_{*}^{\prime}\left(E_{1}\right)$ and satisfies the conditions (i) $\sim$ (iv). This contradicts the assertion (1).

In order to prove $(2)$, it suffices to show that $\left(E_{2} \cdot T\right)=0$. Suppose that $\left(E_{2} \cdot T\right)=1$. Let $\bar{f}: X \rightarrow \bar{Y}$ be a successive contraction of $(-1)$-curves in $F$ such that $\bar{f}_{*}\left(E_{1}\right)$ is a $(-1)$-curve and that $\operatorname{Supp} \bar{f}_{*}(T)$ contains no $(-1)$-curves. If $\bar{f}_{*}\left(A_{3}\right)=0$, then we have a (partial) successive contraction of $(-1)$-curves in $F$, say $f_{1}: X \rightarrow Y_{1}$, such that $f_{1_{*}}\left(E_{1}\right)$ and $f_{1_{*}}\left(A_{3}\right)$ are $(-1)$-curves. Since $f_{1_{*}}\left(A_{1}\right)$ and $f_{1 *}\left(A_{2}\right)$ are $(-2)$-curves, it follows that $f_{1 *}(F)=f_{1 *}\left(A_{1}+A_{2}+2 A_{3}\right)$. This is a contradiction. Hence $\bar{f}_{*}\left(A_{3}\right) \neq 0$. Then $\bar{f}_{*}\left(A_{1}\right)$ and $\bar{f}_{*}\left(A_{2}\right)$ are $(-2)$-curves. Thus $\bar{f}_{*}(F)$ contains a unique $(-1)$-curve $\bar{f}_{*}\left(E_{1}\right)$ and satisfies the conditions (i) (iv). This contradicts the assertion (1). Hence we may assume that $\left(E_{2} \cdot A_{2}\right)=0$.

Q.E.D.

Lemma 3.8. $\Delta$ does not contain $D_{4}$.

Proof. Suppose that $\Delta$ contains $D_{4}$. Then $D_{3}$ is a cross-section of $\Phi$. Put $\Delta=D_{4}+D_{5}+\cdots+D_{i_{1}}$. Let $S_{1}$ be a singular fiber of $\Phi$ containing $D_{0}, D_{1}$ and $D_{2}$. We consider the following three cases separately.

Case 1: $i_{1}=r$. By the count of $\rho(X)$, we know that all singular fibers of $\Phi$ are exhausted by $S_{0}$ and $S_{1}$ and that $S_{1}$ has a unique $(-1)$-curve, say $E_{1}$. Since $E_{1}+D_{0}+D_{1}+D_{2}$ can be contracted to a nonsingular rational curve with selfintersection number zero, it follows that $\left(E_{1} \cdot D\right)=\left(E_{1} \cdot D_{0}\right)=1, a_{0}=2$, and $S_{1}=2\left(E_{1}+D_{0}\right)+D_{1}+D_{2}$. This is a contradiction because $D_{0}$ meets $D_{3}$ which is a cross-section of $\Phi$.

Case 2: $i_{1}=r-1$. Since $D_{r}$ is a cross-section of $\Phi$, there exists a (-1)-curve, say $E_{1}$, in $S_{1}$ such that $\left(E_{1} \cdot D_{r}\right)=1$. Then, by the count of $\rho(X)$, it follows that $S_{1}$ has two (-1)-curves and $\Phi$ has no singular fibers other than $S_{0}$ and $S_{1}$. Let $E_{2}$ be another $(-1)$-curve in $\operatorname{Supp}\left(S_{1}\right)$. Note that $\left(E_{2} \cdot D\right)=\left(E_{2} \cdot D_{0}+D_{1}+D_{2}\right)=1$. 
Suppose that $E_{2}$ meets $D_{0}$. It then follows from Lemma 1.2 that $\left(D_{0}^{2}\right)=-2$. Then $S_{1}=2\left(E_{2}+D_{0}\right)+D_{1}+D_{2}$, and this contradicts the existence of $E_{1}$. This implies that $E_{2}$ meets either $D_{1}$ or $D_{2}$. We may assume $\left(E_{2} \cdot D_{2}\right)=1$. Then $\left(D_{0}^{2}\right)=-2$ by Lemma 1.2. This implies that $\left(E_{1} \cdot D_{1}\right)=1$.

Let $\mu: X \rightarrow \mathbf{F}_{n}$ be a successive contraction of $(-1)$-curves in $S_{0}$ and $S_{1}$ such that $\mu_{*}\left(S_{0}\right)=\mu_{*}\left(D_{4}\right)$ and $\mu_{*}\left(S_{1}\right)=\mu_{*}\left(D_{0}\right)$. Then $n=-a_{3} \geq 2$ and $\left(\mu_{*}\left(D_{r}\right)^{2}\right)=$ $-a_{r}+3 \leq 1$, which contradict Lemma 1.8 .

Case 3: $\quad i_{1} \leq r-2$. Let $S_{2}$ be a singular fiber of $\Phi$ containing $D_{i_{1}+2}, \ldots, D_{r}$. Since $D_{3}$ and $D_{i_{1}+1}$ are cross-sections and by the count of $\rho(X)$, it follows that $S_{1}=S_{2}$ and $S_{1}$ has at most two $(-1)$-curves. Hence $S_{1}$ satisfies the conditions (i) $\sim$ (iv) in Lemma 3.7 with $A:=D_{0}+D_{1}+D_{2}$ and $B:=D_{i_{1}+2}+D_{i_{1}+3}+\cdots+D_{r}$. By Lemma $3.7(1)$, there exist two (-1)-curves $E_{1}$ and $E_{2}$ in $\operatorname{Supp}\left(S_{0}\right)$. Assume that $\left(E_{1} \cdot A\right)=\left(E_{1} \cdot B\right)=1$. By Lemma $3.7(2)$ we may assume that $\left(E_{2} \cdot D_{2}\right)=1$. Since $\left(E_{2} \cdot D\right)=\left(E_{2} \cdot A\right)=1$ and by Lemma 1.2 , it follows that $a_{0}=2$. This is a contradiction because the intersection matrix of $E_{1}+E_{2}+A$ is then not negative definite.

This completes the proof of Lemma 3.8.

Finally, we consider the case where $i_{0} \geq 5$.

Lemma 3.9. Suppose that $i_{0} \geq 5$. Then $i_{0}=5, i_{1}=r$ and there exists a $(-1)$-curve $E$ such that $(E \cdot D)=\left(E \cdot D_{t}\right)=1$, where $t=1$ or 2 . The weighted dual graph of $E+C+D$ is given in Appendix $\mathrm{B}$, where $E_{1}$ is to be read as $C$.

Proof. The component $D_{i_{0}-1}$ is a cross-section of $\Phi$. Let $S_{1}$ be a singular fiber of $\Phi$ containing $D_{0}, \ldots, D_{i_{0}-2}$. As in the proof of Lemma 3.8, we consider the following three cases separately.

Case 1: $i_{1}=r$. By the count of $\rho(X)$, all singular fibers of $\Phi$ are then exhausted by $S_{0}$ and $S_{1}$. Furthermore, $S_{1}$ has a unique (-1)-curve, say $E$. Suppose that $\left(E \cdot D_{0}+D_{3}+\cdots+D_{i_{0}-2}\right)=1$. Since $E+D_{0}+D_{1}+D_{2}+D_{3}+\cdots+D_{i_{0}-2}$ can be contracted to a nonsingular rational curve with self-intersection number zero, the weighted dual graph of $\operatorname{Supp}\left(S_{1}\right)$ is the one in Figure 2. This is a contradiction because $D_{i_{0}-2}$ meets $D_{i_{0}-1}$, which is a cross-section of $\Phi$. Hence $\left(E \cdot D_{1}\right)=1$ or $\left(E \cdot D_{2}\right)=1$. Assume that $\left(E \cdot D_{1}\right)=1$. Since $(E \cdot D)=\left(E \cdot D_{1}\right)=1$, it follows from Lemma 1.2 and the almost minimality of $(X, D)$ that $a_{0}=a_{3}=2$. Then $S_{1}=2\left(E+D_{0}+D_{1}\right)+D_{2}+D_{3}$ and hence $i_{0}=5$. The weighted dual graph of $E+C+D$ is then given in Appendix B, where $E_{1}$ is to be read as $C$.

Case 2: $i_{1}=r-1$. Since $D_{r}$ is a cross-section of $\Phi, S_{1}$ has a $(-1)$-curve, say $E_{1}$, such that $\left(E_{1} \cdot D_{r}\right)=1$. Then $S_{1}$ has another $(-1)$-curve $E_{2}$. By the count of $\rho(X), S_{1}$ has exactly two $(-1)$-curves. Then $\left(E_{2} \cdot D\right)=\left(E_{2} \cdot D_{0}+\right.$ $\left.D_{1}+\cdots+D_{i_{0}-2}\right)=1$. Suppose that $\left(E_{2} \cdot D_{1}\right)=1$. By the argument as in 
Case 1 , we know that $a_{0}=a_{3}=2$ and $S_{1}=2\left(E_{2}+D_{0}+D_{1}\right)+D_{2}+D_{3}$. This contradicts the existence of $E_{1}$. Similarly, $\left(E_{2} \cdot D_{2}\right)=1$ is impossible. Hence $\left(E_{2} \cdot D_{0}+D_{3}+\cdots+D_{i_{0}-2}\right)=1$. Since the multiplicity of $E_{1}$ in $S_{1}$ is equal to one, we can find a birational morphism $f: X \rightarrow X^{\prime}$ which is a successive contraction of $(-1)$-curves in $S_{1}$ starting with the contraction of $E_{2}$ such that $f_{*}\left(D_{0}\right)$ and $f_{*}\left(E_{1}\right)$ are $(-1)$-curves. By the condition on $f$, it follows that $f_{*}\left(D_{1}\right)$ and $f_{*}\left(D_{2}\right)$ are $(-2)$-curves. Hence $f_{*}\left(S_{1}\right)=f_{*}\left(2 D_{0}+D_{1}+D_{2}\right)$, which is a contradiction.

Case 3: $i_{1} \leq r-2$. By the count of $\rho(X), S_{1}$ contains at most two (-1)curves and $\left\{D_{i_{1}+2}, D_{i_{1}+3}, \ldots, D_{r}\right\}$. Put $A:=D_{0}+D_{1}+D_{2}+\cdots+D_{i_{0}-2}$ and $B:=D_{i_{1}+2}+D_{i_{1}+3}+\cdots+D_{r}$. Then $S_{1}$ satisfies the conditions (i) $\sim$ (iv) in Lemma 3.7. By Lemma $3.7(1), \operatorname{Supp}\left(S_{1}\right)$ has two $(-1)$-curves $E_{1}$ and $E_{2}$. Assume that $\left(E_{1} \cdot A\right)=\left(E_{1} \cdot B\right)=1$. By Lemma $3.7(2)$, we may assume that $\left(E_{2} \cdot D_{1}\right)=1$. By arguing as in Case 2, we have a contradiction.

This completes the proof of Lemma 3.9.

Proposition 3.4 is thus proved. The assertion (3) of Theorem 3.1 is then clear. Theorem 3.1 is thus verified.

\section{Cyclic quotient singular points}

Let $(X, D)$ be an almost minimal pair as in Main Theorem. In this section, we treat the case where $P$ has a cyclic quotient singularity. The weighted dual graph of $D$ is given in Figure 11, where $a_{i} \geq 2$ for $i=1,2, \ldots, r$.

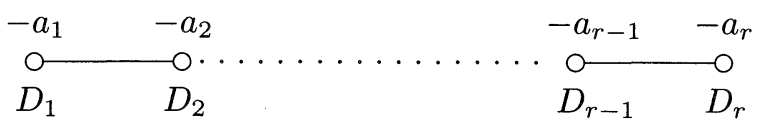

Figure 11

Our main result of this section is the following:

Theorem 4.1. Let $\bar{X}$ be a log del Pezzo surface of rank one with a unique singular point $P$ and let $(X, D)$ be the minimal resolution of $\bar{X}$. Suppose that $P$ is a cyclic quotient singular point. Then we have the following:

(1) If $(X, D) \neq\left(\mathbf{F}_{n}, M_{n}\right)$ then the weighted dual graph of $D$ is given by one of (a), (b) and (c) in Appendix C.

(2) If the weighted dual graph of $D$ is given by one of (a) and (b) in Appendix $\mathrm{C}$, then there exist a $\mathbf{P}^{1}$-fibration $\Phi: X \rightarrow \mathbf{P}^{1}$ and an irreducible component $H$ of $D$ such that $H$ is a cross-section of $\Phi$ and the other irreducible components of $D$ are contained in singular fibers of $\Phi$. Furthermore, $\Phi$ has at most two singular 
fibers.

(3) If the weighted dual graph of $D$ is given by (c) in Appendix $\mathrm{C}$, then there exists a $\mathbf{P}^{1}$-fibration $\Psi: X \rightarrow \mathbf{P}^{1}$ and two irreducible components $H_{1}$ and $H_{2}$ such that $H_{1}$ and $H_{2}$ are cross-sections of $\Psi$ and the other irreducible components of $D$ are contained in singular fibers of $\Psi$. Furtheremore, $\Psi$ has exactly two singular fibers.

(4) All of the types of singularities listed in Appendix $\mathrm{C}$ are realizable.

In what follows, we prove the above theorem.

LEMMA 4.2. With the notation and assumptions as above, either Theorem 4.1 is true or there exists a $(-1)$-curve $E$ such that $(E \cdot D)=1$.

Proof. Let $C$ be an irreducible curve such that $-\left(C \cdot D^{\#}+K\right)$ attains the smallest positive value (cf. $\S 1$ ). If $|C+D+K|=\emptyset$ then, by Lemma 1.5 , it follows that either $C$ is a $(-1)$-curve with $(C \cdot D)=1$ or $(X, D)=\left(\mathbf{F}_{n}, M_{n}\right)$, where $n=-\left(D^{2}\right) \geq 2$.

Suppose that $|C+D+K| \neq \emptyset$. By Lemma 1.3 and its proof, we know that either $C+D+K \sim 0$, or $C+K \sim 0$ and $D$ is a rod of $(-2)$-curves. If the latter case takes place, the assertion follows from [9, Lemma 3]. Suppose that $C+D+K \sim 0$. Since $|C+K|=|-D|=\emptyset, C$ is a nonsingular rational curve by [6, Lemma 2.1.3]. Furthermore, $(C \cdot D)=-(C \cdot C+K)=2$ and $\left(D_{i} \cdot C+D-D_{i}\right)=-\left(D_{i} \cdot K+D_{i}\right)=2$ for any irreducible component $D_{i}$ of $D$.

If $\left(C \cdot D_{i}\right)=2$ for some $1 \leq i \leq r$, then $D$ is irreducible and the configuration of $C+D$ is one of the next two in Figure 12 .
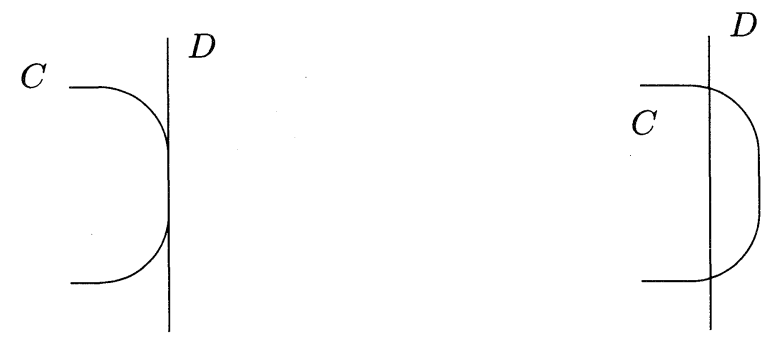

Figure 12

Since $D$ is irreducible, $\rho(X)=2$. Hence $(X, D)=\left(\mathbf{F}_{n}, M_{n}\right)\left(n=-\left(D^{2}\right) \geq 2\right)$. Let $\ell$ be a fiber of the ruling on $\mathbf{F}_{n}$. Then $C \sim M_{n}+(n+2) \ell$ because $C+D \sim-K_{\mathbf{F}_{n}}$. We then have

$$
-\left(C \cdot D^{\#}+K_{\mathbf{F}_{n}}\right)=\left(M_{n}+(n+2) \ell \cdot\left(2-\frac{n-2}{n}\right) M_{n}+(n+2) \ell\right)
$$




$$
\begin{aligned}
& =n+2+\frac{2(n+2)}{n} \\
& >4
\end{aligned}
$$

because $n \geq 2$. On the other hand,

$$
-\left(\ell \cdot D^{\#}+K_{X}\right)=2-\frac{n-2}{n} \leq 2 .
$$

This contradicts the hypothesis that $-\left(C \cdot D^{\#}+K\right)$ attains the smallest positive value. Hence $D$ is reducible and $\left(C \cdot D_{1}\right)=\left(C \cdot D_{r}\right)=1$.

Suppose that $C+D$ is not a divisor of normal crossings. Then $r=2$ and the configuration of $C+D$ is given as in Figure 13 .

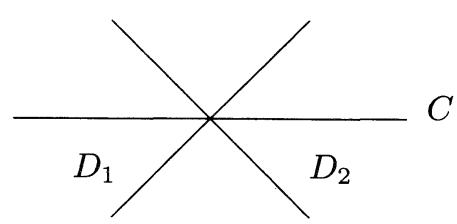

Figure 13

Since $C$ is a nonsingular rational curve, we have $\left(C^{2}\right) \geq-1$ by Lemma 1.2. If $\left(C^{2}\right) \geq 0$ then the Riemann-Roch theorem yields that

$$
\operatorname{dim}|C| \geq\left(C^{2}\right)+1 \geq 1
$$

This will also imply that $|C|$ is base point free. Hence there exists a member $C^{\prime}$ of $|C|$ such that $C^{\prime}$ is irreducible and $C^{\prime}+D$ is an SNC-divisor. Suppose that $C$ is a (-1)-curve. Since $K \sim-\left(C+D_{1}+D_{2}\right)$, we have

$$
\left(K^{2}\right)=\left(C+D_{1}+D_{2}\right)^{2}=\left(C^{2}\right)+\left(D_{1}^{2}\right)+\left(D_{2}^{2}\right)+6 \leq 1 .
$$

Hence $\rho(X)=10-\left(K^{2}\right) \geq 9$. On the other hand, $\rho(X)=3$ because $r=2$ and $\rho(X)=r+1$. This is a contradiction.

Hence we have the following:

Lemma 4.3. Suppose that $C+D+K \sim 0$ and $P$ is not a rational double point. Then by taking a suitable member of $|C|$, we may assume that $C+D$ is an SNC-divisor.

Proof of Lemma 4.2 CONTINUEd. Since $D$ is reducible $X$ is not relatively minimal. So the set $\Sigma$ of all $(-1)$-curves on $X$ is not empty. If $E$ is in $\Sigma-\{C\}$ 
then

$$
0=(E \cdot C+D+K)=(E \cdot C)+(E \cdot D)-1
$$

and hence $(E \cdot D)=1$ and $(E \cdot C)=0$ by Lemma 1.2. Lemma 4.2 is true. So we may assume that $\Sigma=\{C\}$. Let $\Phi$ be any $\mathbf{P}^{1}$-fibration on $X$. Then by Lemma 1.2 and the count of $\rho(X), \Phi$ has exactly one singular fiber $S_{0}$, Supp $S_{0}=\operatorname{Supp}\left(C+D-D_{i}\right)$ where $i=1$ or $r$, and $D_{i}$ is a cross-section. Cleary, Theorem 4.1 is true.

This completes the proof of Lemma 4.2.

If $X$ is relatively minimal then $(X, D)=\left(\mathbf{F}_{n}, M_{n}\right)$ for some $n \geq 2$. So, in the subsequent arguments, we assume that $X$ is not relatively minimal. Then we may assume that there exists a $(-1)$-curve $E$ such that $(E \cdot D)=1$ by Lemma 4.2. By the Hodge index theorem and the fact that $\rho(X)=\#(D)+1$, the intersection matrix of $E+D$ is neither negative definite nor negative semi-definite. Hence there exist an effective divisor $\Delta_{0}$ with $\operatorname{Supp}\left(\Delta_{0}\right) \subset \operatorname{Supp}(D)$ and an integer $e>0$ such that $S_{0}:=e E+\Delta_{0}$ defines a $\mathbf{P}^{1}$-fibration $\Phi: X \rightarrow \mathbf{P}^{1}$. Put $\Delta:=\left(\Delta_{0}\right)_{\text {red }}=$ $D_{i}+D_{i+1}+\cdots+D_{j}(i<j)$. The weighted dual graph of $C+D$ looks like that of Figure 1. Hereafter in the present section, we retain this situation.

Suppose that $i=1$. Then $j<r$ and $D_{j+1}$ is a cross-section of $\Phi$. Assume that $i=1$ and $j=r-1$. Then $\# S_{0}=r$. Since $\rho(X)=r+1=\left(\# S_{0}-1\right)+2$, it follows that $\Phi$ has no singular fibers other than $S_{0}$. The weighted dual graph of $E+D$ is then given by (a) in Appendix C. Assume that $i=1$ and $j<r-1$. Let $S_{1}$ be a singular fiber of $\Phi$ containing $D_{j+2}, \ldots, D_{r}$. By the count of $\rho(X), S_{1}$ has a unique $(-1)$-curve, say $E_{1}$. Furthermore, $\Phi$ has no singular fibers other than $S_{0}$ and $S_{1}$. The weighted dual graph of $E+D+E_{1}$ is then given by (b) in Appendix C.

Next suppose that $i>1$. If $j=r$ then relabel the $D_{i}$ in $\Delta$ in the reveresed order. Then the argument in the case $i=1$ is used to determine the weighted dual graph of $E+D$ (or $E+D+E_{1}$ ). Thus, in the case $i=1$ or $j=1$, the assertion (2) of Theorem 4.1 holds. So, we assume that $i>1$ and $j<r$ in the subsequent arguments. Then the following assertion holds.

LEMMA 4.4. With the notation and assumptions as above, we have $i>2$ or $j<r-1$.

Proof. Suppose that $i=2$ and $j=r-1$. Then $D_{1}$ and $D_{r}$ are crosssections of $\Phi$. By the count of $\rho(X)$ and Lemma 1.1 (2), there exists another singular fiber $S_{1}=F_{0}+F_{1}$, where $F_{0}$ and $F_{1}$ are $(-1)$-curves. We may assume that $\left(F_{1} \cdot D_{1}+D_{r}\right) \leq 1$. Then we reach a contradiction to Lemma 1.2 or the fact that $\rho(X)=\#(D)+1$.

Q.E.D.

By virtue of Lemma 4.4, we may assume that $i>2$ in the subsequent arguments. Let $S_{1}$ be a singular fiber of $\Phi$ containig $D_{1}, \ldots, D_{i-2}$ and let $S_{2}$ be a 
singular fiber of $\Phi$ containing $D_{j+2}, D_{j+3}, \ldots, D_{r}$ if $j<r-1$.

LEMma 4.5. With the notation and the assumptions as above, the following assertions hold:

(1) If $j<r-1$ then $S_{1}=S_{2}$.

(2) The $\mathbf{P}^{1}$-fibration $\Phi$ has no singular fibers other than $S_{0}$ and $S_{1}$.

(3) $S_{1}$ has exactly two (-1)-curves.

Proof. (1) Suppose to the contrary that $S_{1} \neq S_{2}$. Then $S_{1}$ (resp. $S_{2}$ ) has a $(-1)$-curve $E_{1}\left(\right.$ resp. $\left.E_{2}\right)$ such that $\left(E_{1} \cdot D_{j+1}\right)=1\left(\operatorname{resp} .\left(E_{2} \cdot D_{i-1}\right)=1\right)$. Since the multiplicity of $E_{i}$ in $S_{i}(i=1,2)$ is then equal to one, $S_{1}$ and $S_{2}$ have at least two (-1)-curves each. We have

$$
\left(\# S_{0}-1\right)+\left(\# S_{1}-1\right)+\left(\# S_{2}-1\right) \geq r
$$

This contradicts $\rho(X)=r+1$.

(2) Note that $\left(\# S_{0}-1\right)+\left(\# S_{1}-1\right) \geq r-2$. The $\mathbf{P}^{1}$-fibration $\Phi$ therefore has at most one singular fiber other than $S_{0}$ and $S_{1}$. Suppose that $\Phi$ has another singular fiber, say $S_{2}$. Lemma 1.1 (2) implies that the singular fiber $S_{2}$ consists of two $(-1)$-curves, say $F_{0}$ and $F_{1}$. By Lemma 1.2 , we may assume that $\left(F_{0}\right.$. $\left.D_{i-1}\right)=\left(F_{1} \cdot D_{j+1}\right)=1$ and $\left(F_{1} \cdot D_{i-1}\right)=\left(F_{0} \cdot D_{j+1}\right)=0$. By the count of $\rho(X)$, $S_{1}$ has a unique $(-1)$-curve, say $E_{1}$. Then there exists a birational morphism $f: X \rightarrow \mathbf{F}_{n}$ which is a successive contraction of $(-1)$-curves in $S_{0}, S_{1}$ and $S_{2}$ such that $f_{*}\left(S_{0}\right)=f_{*}\left(D_{j}\right), f_{*}\left(S_{1}\right)=f_{*}\left(D_{j+2}\right)$ and $f_{*}\left(S_{2}\right)=f_{*}\left(F_{1}\right)$. We know that $n=-a_{j+1} \geq 2$ and $f_{*}\left(D_{j+1}\right)=M_{n}$. On the other hand, since $S_{0}$ and $S_{1}$ have only one $(-1)$-curve each, $\left(f_{*}\left(D_{i-1}\right)^{2}\right)=\left(D_{i-1}^{2}\right)+3 \leq 1$. This contradicts Lemma 1.8 .

(3) By the assertion (2) and the count of $\rho(X)$, the assertion (3) is clear.

Q.E.D.

Put $A=D_{1}+\cdots+D_{i-2}(\neq 0)$ and $B=D_{j+2}+\cdots+D_{r}$ if $j \leq r-2$. Let $E_{1}$ be a $(-1)$-curve in $\operatorname{Supp}\left(S_{1}\right)$ such that $\left(A \cdot E_{1}\right)=\left(E_{1} \cdot D_{r}\right)=1$ if $j=r-1$ and $\left(A \cdot E_{1}\right)=\left(B \cdot E_{1}\right)=1$ if $j \leq r-2$. We prove the following:

LEMMA 4.6. With the notation and assumptions as above, we have

$$
\left(E_{1} \cdot D_{1}\right)=\left(E_{1} \cdot D_{r}\right)=1
$$

Proof. First, we consider the case $j=r-1$. The multiplicity of $E_{1}$ in $S_{1}$ is then equal to one because $D_{r}$ is a cross-section of $\Phi$. By Lemma 4.5 (3), we can find a birational morphism $f: X \rightarrow Y$ which is a successive contraction of $(-1)$ curves in $S_{1}$ such that $f_{*}\left(D_{i-2}\right)$ and $f_{*}\left(E_{1}\right)$ are all $(-1)$-curves in $\operatorname{Supp} f_{*}\left(S_{1}\right)$. Since the multiplicities of $f_{*}\left(D_{i-2}\right)$ and $f_{*}\left(E_{1}\right)$ in $f_{*}\left(S_{1}\right)$ are equal to one and Supp $f_{*}\left(\left(S_{1}\right)_{\text {red }}-D_{i-2}-E_{1}\right)$ is a linear chain, the weighted dual graph of $f_{*}\left(S_{1}\right)$ 
looks like that of Figure 8. By Lemma 4.5 (3), the number of the fundamental points of $f^{-1}$ is equal to one. Let it be $P^{\prime}$. Furthermore, we know that $P^{\prime} \in f_{*}\left(D_{i-2}\right)$ and $P^{\prime} \notin f_{*}\left(E_{1}\right)$. So, by the condition on $f,\left(E_{1} \cdot D_{1}\right)=1$. In this case, the assertion is thus verified.

Next consider the case $j \leq r-2$. Let $E_{2}$ be a $(-1)$-curve in $S_{1}$ other than $E_{1}$. We consider the case $\left(E_{2} \cdot A\right)=1$. The case $\left(E_{2} \cdot B\right)=1$ can be treated in a similar way. Since $\left(E_{1} \cdot A\right)=\left(E_{1} \cdot B\right)=1$ and $A, B \neq 0$, the multiplicity of $E_{1}$ in $S_{1}$ $>1$. Let $h: X \rightarrow \bar{X}_{1}$ be a successive contraction of $(-1)$-curves in $E_{2}+A$, which starts with the contraction of $E_{2}$, such that $h_{*}\left(E_{1}\right)$ is a $(-1)$-curve and $h_{*}\left(E_{2}+A\right)$ has no $(-1)$-curves. Since $S_{1}$ has two $(-1)$-curves and $\left(S_{1}\right)_{\text {red }}-E_{1}-E_{2}$ has two connected components $A$ and $B$, such a morphism $h$ exists. Then $h_{*}\left(E_{2}+A\right) \neq 0$. We prove the following:

Claim. $\operatorname{Supp} h_{*}\left(S_{1}\right)$ is a linear chain.

Let $A_{k}$ (resp. $B_{\ell}$ ) be an irreducible component of $h_{*}(A)$ (resp. $h_{*}(B)$ ) which $h_{*}\left(E_{1}\right)$ meets. Suppose to the contrary that $\operatorname{Supp} h_{*}\left(S_{1}\right)$ is not a linear chain. $A_{k}$ is not a terminal component of $\operatorname{Supp} h_{*}(A)$ or $B_{\ell}$ is not a terminal component of Supp $h_{*}(B)$. We may assume that the first case takes place. The latter case can be treated similarly.

Let $h_{2}: \bar{X}_{1} \rightarrow \bar{X}_{2}$ be a successive contraction of $(-1)$-curves in $h_{*}\left(E_{1}+B\right)$ such that $h_{2 *}\left(A_{k}\right)$ is a $(-1)$-curve. Then $\operatorname{Supp}\left(h_{2} \circ h\right)_{*}\left(S_{1}\right)$ is a linear chain, i.e., $\left(h_{2} \circ h\right)_{*}\left(E_{1}+B\right)=0$. Furthermore, the multiplicity of $h_{2 *}\left(A_{k}\right)$ in $\left(h_{2} \circ h\right)_{*}\left(S_{1}\right)$ is then equal to one. This is a contradiction because $h_{2 *}\left(A_{k}\right)$ is not a terminal component of $\operatorname{Supp}\left(h_{2} \circ h\right)_{*}\left(S_{1}\right)$ by the condition on $h_{2}$.

Hence $\operatorname{Supp} h_{*}\left(S_{1}\right)$ is a linear chain.

Since $\operatorname{Supp} h_{*}\left(S_{1}\right)$ is a linear chain by Claim and contains a unique (-1)curve $h_{*}\left(E_{1}\right), h_{*}\left(S_{1}\right)$ contains exactly two multiplicity 1 components: they are end components of $\operatorname{Supp} h_{*}\left(S_{1}\right)$. Hence we know that $\left(E_{1} \cdot B\right)=\left(E_{1} \cdot D_{r}\right)=1$. In order to prove Lemma 4.6 , it suffices to show that $\left(E_{1} \cdot A\right)=\left(E_{1} \cdot D_{1}\right)=1$. Suppose that $\left(E_{1} \cdot A\right)=\left(E_{1} \cdot D_{s}\right)=1$ for $s \geq 2$. Let $t$ be an integer such that $\left(E_{2} \cdot A\right)=\left(E_{2} \cdot D_{t}\right)=1$. If $s \leq t$ then the multiplicity of $h_{*}\left(D_{s}\right)$ in $h_{*}\left(S_{1}\right)$ is equal to one. This is a contradiction because $h_{*}\left(D_{s}\right)$ is not a terminal component of Supp $h_{*}\left(S_{1}\right)$. Hence $s>t$. The configuration of $S_{1}+D_{i-1}+D_{j+1}$ is then given as in Figure 14. Put $\bar{D}_{m}:=h_{*}\left(D_{m}\right)$ for $m=s, s+1, \ldots, i-2, j+1, j+2, \ldots, r$ and put $\bar{S}_{i}:=h_{*}\left(S_{i}\right)$ for $i=0,1$. By Claim (2) we have $h_{*}\left(E_{2}+A\right)=\sum_{m=k}^{i-2} \bar{D}_{m}$. Let $\tilde{h}: \bar{X}_{1} \rightarrow \mathbf{F}_{n}$ be a successive contraction of $(-1)$-curves in $\bar{S}_{0}$ and $\bar{S}_{1}$ such that $\tilde{h}_{*}\left(\bar{S}_{0}\right)=(\tilde{h} \circ h)_{*}\left(D_{j}\right)$ and $\tilde{h}_{*}\left(\bar{S}_{1}\right)=\tilde{h}_{*}\left(\bar{D}_{j+2}\right)$. It then follows that $n=-\left(D_{j+1}^{2}\right) \geq$ $2, M_{n}=(\tilde{h} \circ h)_{*}\left(D_{j+1}\right)$ and $\tilde{h}_{*}\left(\bar{D}_{i-1}\right)^{2}=\left(D_{i-1}^{2}\right)+2 \leq 0$. This contradicts Lemma 1.8 . 


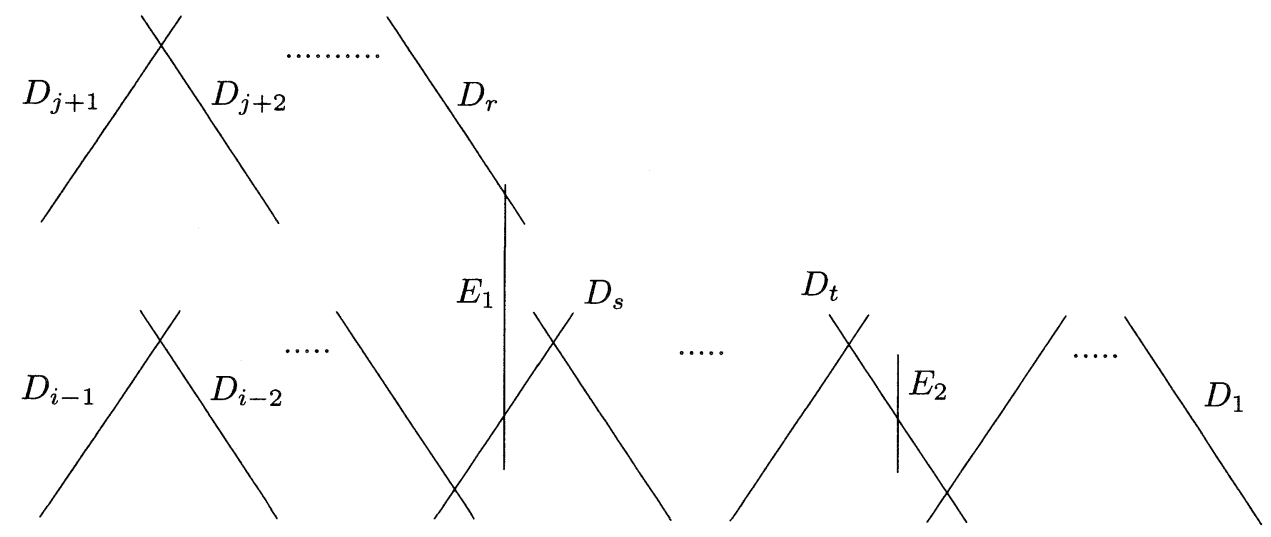

Figure 14

This completes the proof of Lemma 4.6.

In what follows, we shall precisely describe the weighted dual graph of $\operatorname{Supp}\left(S_{1}\right)$, when $i>2$ and $j<r$.

Suppose that $B=\emptyset$, i.e., $j=r-1$. Let $\mu: X \rightarrow X^{\prime}$ be a successive contraction of (-1)-curves in $E_{2}+A$ such that $\mu_{*}\left(D_{i-2}\right)$ becomes a (-1)-curve. The configuration of $\operatorname{Supp} \mu_{*}\left(S_{1}\right)$ is then given as in Figure 15, where $m$ is the number of irreducible components of $\operatorname{Supp} \mu_{*}\left(S_{1}\right)$. Note that, by the condition on $\mu,\left(\mu_{*}\left(D_{i-1}\right)^{2}\right)=\left(D_{i-1}^{2}\right)$.

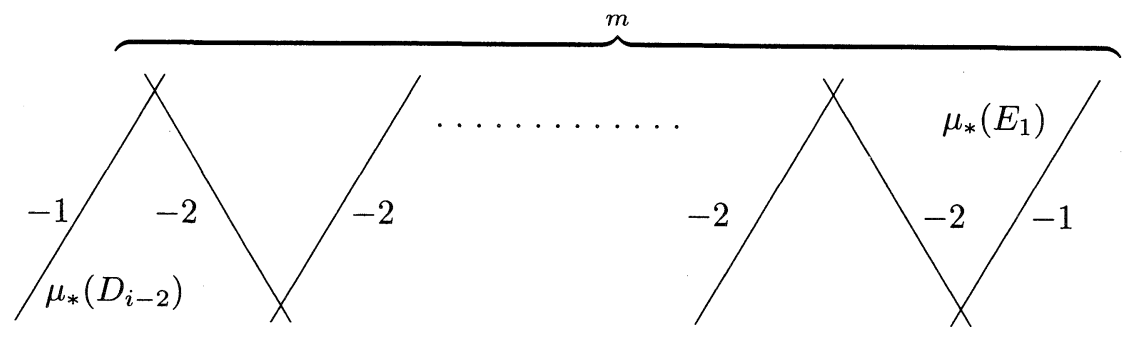

Figure 15

Let $\mu^{\prime}: X^{\prime} \rightarrow \mathbf{F}_{n}$ be a successive contraction of $(-1)$-curves in $\mu_{*}\left(S_{0}\right)$ and $\mu_{*}\left(S_{1}\right)$ such that $\left(\mu^{\prime} \circ \mu\right)_{*}\left(S_{0}\right)=\left(\mu^{\prime} \circ \mu\right)_{*}\left(D_{j}\right)$ and $\left(\mu^{\prime} \circ \mu\right)_{*}\left(S_{1}\right)=\left(\mu^{\prime} \circ \mu\right)_{*}\left(E_{1}\right)$. Then $n=a_{j+1} \geq 2, M_{n}=\left(\mu^{\prime} \circ \mu\right)_{*}\left(D_{j+1}\right)$ and $\left(\mu^{\prime} \circ \mu\right)_{*}\left(D_{i-1}\right)^{2}=m-a_{i-1}$. Lemma 1.7 yields that

$$
m-a_{i-1}=\left(\mu^{\prime} \circ \mu\right)_{*}\left(D_{i-1}\right)^{2}=n=a_{j+1} .
$$


The weighted dual graph of $\operatorname{Supp}\left(S_{1}\right)$ is then given as in Figure 16, where for the definition of the subgraph denoted by the encircled $\tilde{T}_{\gamma}(\gamma \geq 0)$, see Appendix C.

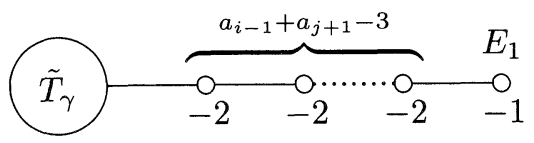

Figure 16

Hence the weighted dual graph of $E+E_{1}+E_{2}+D$ is given by (c) in Appendix C.

Next suppose that $B \neq \emptyset$. Then there exists a successive contraction of $(-1)$ curves in $S_{1}$, say $\mu: X \rightarrow \tilde{X}$, which starts with the contraction of $E_{1}$, such that $\mu_{*}\left(E_{2}\right)$ and one of $\mu_{*}\left(D_{i-2}\right)$ and $\mu_{*}\left(D_{j+2}\right)$ are all $(-1)$-curves in $\operatorname{Supp} \mu_{*}\left(S_{1}\right)$. Indeed, if such a morphism $\mu$ does not exist, then one can find a successive contraction of $(-1)$-curves in $S_{1}$, say $f: X \rightarrow Y$, such that $f_{*}\left(D_{j+2}\right) \neq 0, f_{*}\left(D_{i-2}\right) \neq 0$ and $f_{*}\left(S_{1}\right)$ has a unique $(-1)$-curve $f_{*}\left(E_{2}\right)$. By Lemma 4.6, $\operatorname{Supp}\left(f_{*}\left(S_{1}\right)_{\text {red }}-f_{*}\left(E_{2}\right)\right)$ is a linear chain. There exists a birational morphism $g: Y \rightarrow \mathbf{F}_{\ell}$ which is a successive contraction of $(-1)$-curves in $f_{*}\left(S_{0}\right)$ and $f_{*}\left(S_{1}\right)$ such that $(g \circ f)_{*}\left(S_{0}\right)=$ $(g \circ f)_{*}\left(D_{j}\right)$ and $(g \circ f)_{*}\left(S_{1}\right)=(g \circ f)_{*}\left(D_{j+2}\right)$. It then follows that $\ell=a_{j+1} \geq 2$, $M_{\ell}=(g \circ f)_{*}\left(D_{j+1}\right)$ and $(g \circ f)_{*}\left(D_{i-1}\right)^{2}=-a_{i-1}+2 \leq 0$. This contradicts Lemma 1.8. So, by arguing as in the case $B=\emptyset$, the weighted dual graph of $\operatorname{Supp}\left(S_{1}\right)$ is given as in Figure 17, where for the definition of the subgraphs denoted by the encircled $T_{\beta}(\beta \geq 1)$ and $\tilde{T}_{\gamma}(\gamma \geq 0)$, see Appendix C.

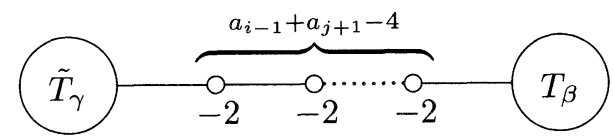

Figure 17

Hence the weighted dual graph of $E+E_{1}+E_{2}+D$ is given by (c) in Appendix C.

Thus, the assertion (3) of Theorem 4.1 is verified. The assertion (4) of Theorem 4.1 is then clear. Theorem 4.1 is thus verified.

REMARK 4.7. Assume that the weighted dual graph of $D$ is given by (c) in Appendix C. We can construct such a pair $(X, D)$ starting from the Hirzebruch surface $\mathbf{F}_{2}$ of degree 2 . 


\section{Appendix}

\section{A. The case of type $E_{n}$}

In the following list of configurations, a solid line stands for a component of $\operatorname{Supp}(D)$; the self-intersection number -2 of a $(-2)$-curve of $D$ is omitted; a line with $*$ on it is not contained in any fiber of the vertical $\mathbf{P}^{1}$-fibration $\Phi: X \rightarrow \mathbf{P}^{1}$.

(Type $E_{6}$ )

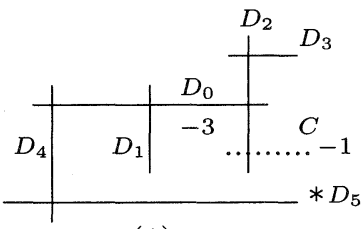

(1)

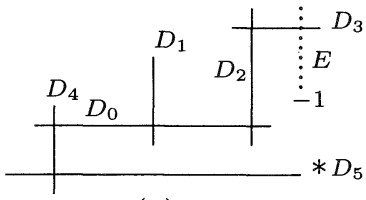

(2)

(Type $E_{7}$ )

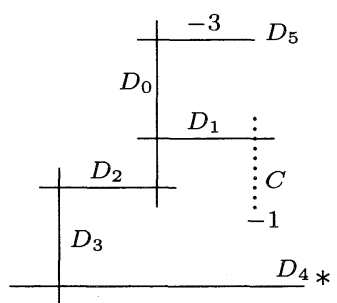

(3)

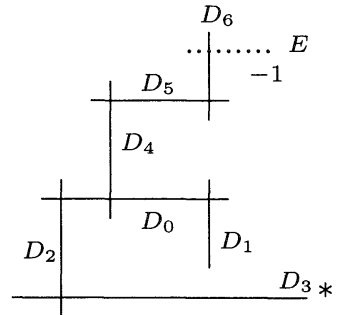

(4)

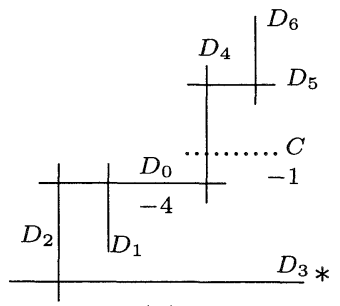

(5)

(Type $E_{8}$ )

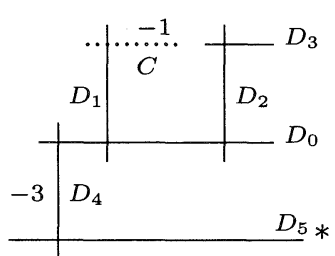

(6)

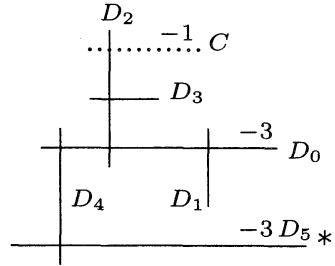

(7)

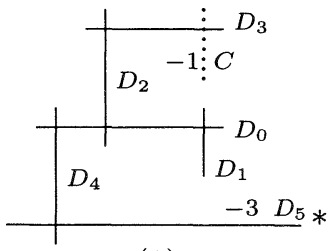

(8) 


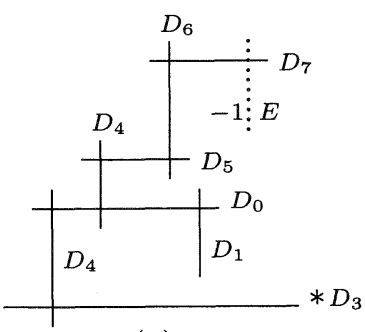

(9)

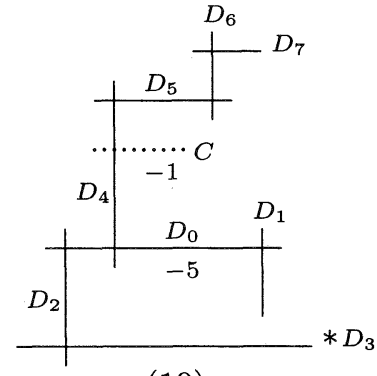

$(10)$

Figure 18

B. The case of type $D$
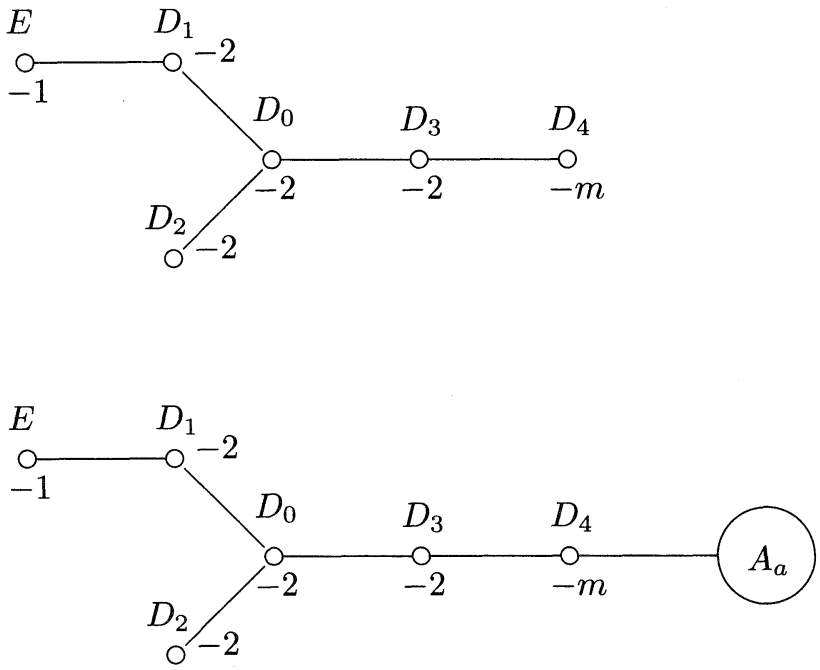

Figure 19

In the above list of the weighted dual graphs, $m \geq 2$ and the subgraph denoted by the encircled $A_{a}(a \geq 1)$ is given as in Figure 1 , where $m_{1} \geq 2, m_{i} \geq 1$ for $2 \leq i \leq a$ and $E$ is to be read as $E_{1}$. 


\section{Cyclic quotient singularities}

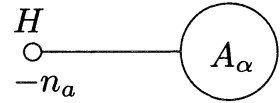

(a)

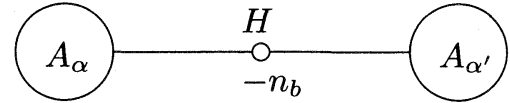

(b)

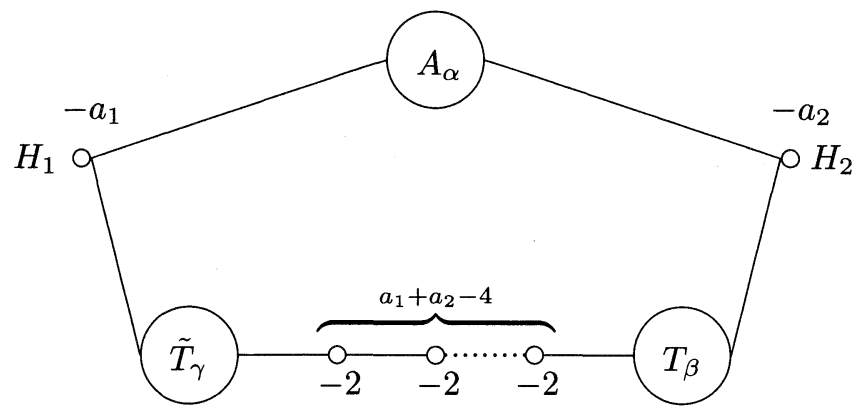

(c)

Figure 20
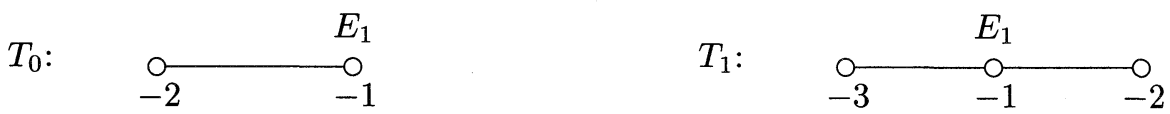

$$
T_{\beta}(\beta \geq 2):
$$

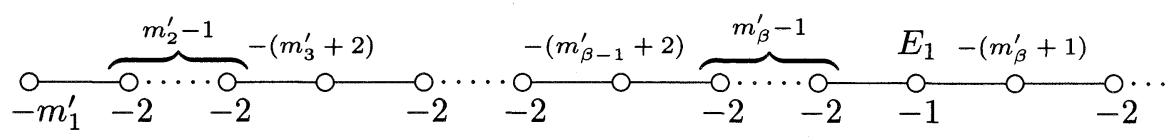

$$
\cdots \cdots \cdots \overbrace{-2}^{-\left(m_{2}^{\prime}+2\right)} \overbrace{-2 \quad-2}^{m_{0}^{\prime}-3}
$$




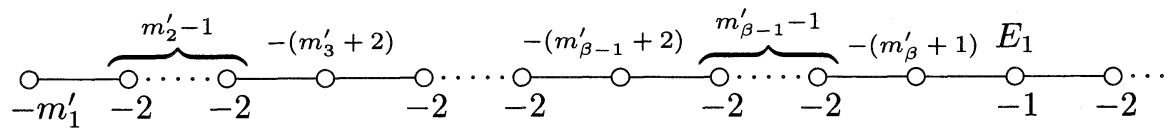

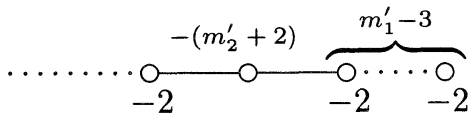

$(\beta$ : odd $)$

Figure 21

We omit the case where $(X, D)=\left(\mathbf{F}_{n}, M_{n}\right)$. In the above list of the weighted dual graphs, the subgraph denoted by the encircled $A_{\alpha}(\alpha \geq 1)$ is given as in Figure 1 , where $m_{1} \geq 3$ and $m_{i} \geq 1$ for $2 \leq i \leq \alpha$, the subgraph denoted by the encircled $T_{\beta}(\beta \geq 0)$ is given as in the above Figure 21, where $m_{1}^{\prime} \geq 3$ and $m_{i}^{\prime} \geq 1$ for $2 \leq i \leq \beta$, and the subgraph denoted by the encircled $\tilde{T}_{\gamma}$ is given $S_{\gamma}$ as in Figure 4 , where $m_{i}$ (resp. $E$ ) is to be read as $\tilde{m}_{i}$ (resp. $\left.E_{2}\right), \tilde{m}_{1} \geq 3$ and $\tilde{m}_{i} \geq 1$ for $2 \leq i \leq \gamma$.

\section{References}

[1] E. Brieskorn, Rationale Singularitäten komplexer Flächen, Invent. Math., 4 (1968), 336358.

[2] R.V. Gurjar and D.-Q. Zhang, $\pi_{1}$ of smooth points of a log del Pezzo surface is finite: I, J. Math. Sci. Tokyo, 1 (1994), 137-180.

[ 3 ] Y. Kawamata, K. Matsuka and K. Matsuki, Introduction to the minimal model program, Adv. Stud. Pure Math, 10 (1987), 283-360.

[4] H. Kojima, Almost minimal embeddings of quotient singular points into rational surfaces, J. Math. Kyoto Univ., 38 (1998), 77-99.

[ 5 ] J. Kollár, et al., Flips and abundance for algebraic threefolds, Astérisque, 211 (1992).

[6] M. Miyanishi, Non-complete algebraic surfaces, Lecture Notes in Math., No. 857, BerlinHeiderberg-New York, Springer, 1981.

[7] M. Miyanishi and S. Tsunoda, Non-complete algebraic surfaces with logarithmic Kodaira dimension $-\infty$ and with non-connected boundaries at infinity, Japan. J. Math., 10 (1984), 195-242.

[8] M. Miyanishi and S. Tsunoda, Logarithmic del Pezzo surfaces of rank one with nonconnected boundaries at infinity, Japan. J. Math., 10 (1984), 271-319

[9] M. Miyanishi and D.-Q. Zhang, Gorenstein log del Pezzo surfaces of rank one, J. Algebra, 118 (1988), 63-84.

[10] D.-Q. Zhang, On Iitaka surfaces, Osaka J. Math., 24 (1988), 417-460.

[11] D.-Q. Zhang, Logarithmic del Pezzo suraces of rank one with contractible boundaries, Osaka J. Math., 25 (1988), 461-497.

[12] D.-Q. Zhang, Logarithmic del Pezzo surfaces with rational double and triple singular points, Tohoku Math. J., 41 (1989), 399-452. 
Department of Mathematics Graduate School of Science OSAKA UNIVERSITY

JAPAN 